\title{
Long-wave theory of bounded two-layer films with a free \\ liquid-liquid interface: Short- and long-time evolution
}

\author{
D. Merkt, A. Pototsky and M. Bestehorn \\ Lehrstuhl für Theoretische Physik II \\ Brandenburgische Technische Universität Cottbus \\ Erich-Weinert-Straße 1, \\ D-03046 Cottbus, Germany \\ e-mail: merkt@physik.tu-cottbus.de \\ U. Thiele \\ Max-Planck-Institut für Physik komplexer Systeme \\ Nöthnitzer Straße 38, \\ D-01187 Dresden, Germany
}




\begin{abstract}
We consider two-layers of immiscible liquids confined between an upper and a lower rigid plate. The dynamics of the free liquid-liquid interface is described for arbitrary amplitudes by a single evolution equation derived from the basic hydrodynamic equations using long-wave approximation. After giving the evolution equation in a general way, we focus on interface instabilities driven by gravity, thermocapillary and electrostatic fields. First, we study the linear stability discussing especially the conditions for destabilizing the system by heating from above or below. Second, we use a variational formulation of the evolution equation based on an energy functional to predict metastable states and the long-time pattern morphology (holes, drops or maze structures). Finally, fully nonlinear three-dimensional numerical integrations are performed to study the short- and longtime evolution of the evolving patterns. Different coarsening modes are discussed and long-time scaling exponents are extracted.
\end{abstract}




\section{INTRODUCTION}

The study of stability properties and pattern formation in thin films is still an important and not fully explored challenge in fluid dynamics. Reorganization processes of such films have remarkable applications in chemical engineering, biological systems, or semiconductor industry. Besides industrial aspects the computational advantage of studying thin films is obvious since one equation for the surface is often sufficient to capture the basic dynamics. Due to the increase of computer power, pattern formation in systems far from equilibrium can be investigated in more detail. This leads to consideration of more and more complex systems which may show a rich variety of bifurcations and allows for a more realistic modelling of fluid phenomena.

Here, we consider thin two-layer films bounded by an upper and a lower rigid plate. Using lubrication approximation a general long-wave interface evolution equation is derived that is valid for arbitrary amplitudes of interface deflections. Pattern formation under the influence of gravity, thermocapillarity and electrostatic forces is analysed.

Lubrication or long-wave approximation is used for more than 100 years to describe the evolution of thin films ${ }^{1}$. In one-layer systems with a free interface the dynamics of the surrounding gas is normally neglected and solely the liquid determines the interface evolution. A simplified equation for the evolution of the profile of the surface can be derived from the basic hydrodynamic equations because the velocity is enslaved to the thickness profile. Several mechanisms are known to destabilize an initially flat surface. A survey of long-wave instabilities in one-layer systems is given by OrON ET AL $\stackrel{2}{*}$. A prominent example is the destabilization and subsequent evolution of a non-flat surface profile due to Marangoni flow caused by heating from below. It was first studied by SCRIVEn and STERLING ${ }^{3}$ and classified 
by Goussis and Kelly $\underline{\underline{4}}^{4}$ as the S-mode instability. The second mode, called P-mode, is a short-wave instability without surface deflection and will not concern us here. However, see Golovin ET AL.$\underline{\underline{5}}$ for a study of the interaction between short- and long-wave mode. The linear and nonlinear behavior for an unstable thin liquid layer heated from below is studied by Burelbach et al. $\underline{\underline{6}}$. Deissler and Oron $\mathrm{N}^{\underline{7}}$ show the stabilizing effect of thermocapillarity for a thin film at the underside of a cooled horizontal plate which is gravitationally or Rayleigh-Taylor (RT) unstable. The normally used linear dependence of surface tension on temperature (linear Marangoni effect) is replaced by ORON and RosenAU $\underline{\underline{8}}$ by a quadratic one, thereby inhibiting true film rupture. Three-dimensional simulations of the linear Marangoni effect are done by ORON ${ }^{\underline{9}}$ and for a wetting film by BESTEHORN ET AL. $\stackrel{10}{\text {. }}$ The former work concentrates on the evolution towards film rupture whereas the latter system allows to explain the preference of drops or holes in dependence of the film thickness. It also gives scaling exponents for the long-time coarsening. In a recent work THIELE and KNOBLOCH ${ }^{11}$ show that the rich bifurcation structure for drop solutions on a horizontal substrate is destroyed even by a rather small inclination of the substrate.

Mathematically related to thin heated films are ultrathin free surface films on horizontal substrates as first studied in long-wave approximation by RUCKENSTEIN and JAIN ${ }^{12}$. These films may be unstable and dewet due to effective molecular interactions that are incorporated into the governing equations in form of an additional pressure term. This so-called disjoining pressure was introduced by DeRJAGuin ET AL. (for an overview see $e^{13}$ ). In the simplest case it results from the apolar London-van der Waals dispersion forces 12 . Open questions regarding dewetting in one-layer systems are summarized in Ref. $\underline{\underline{14}}$. Here, we will use a stabilizing van der Waals interaction to avoid 'true' film rupture for a heated film吕. The evolution of unstable thin films has a general characteristics that is found as well for thin 
heated films as also for ultrathin dewetting films. One distinguishes between a short-time and a long-time behavior. First, the flat film evolves into a large amplitude pattern whose typical length scale can normally be determined by linear considerations. Often, this stage is called initial film rupture although the film may not rupture totally, but an ultrathin film remains at the 'dry' parts. The long-time behavior is characterized by an ongoing coarsening towards patterns of longer and longer horizontal spatial scales.

Evidently, long-wave approximation is not only applicable for single liquid layers on a solid substrate. The approach can be naturally extended towards systems characterized by more than one layer. Taking, for example, two layers of immiscible liquids on a solid horizontal substrate in a gas atmosphere yields a pair of coupled evolution equations for the liquid-liquid and the liquid-gas interfaces. Such a system in presence of a surfactant and an evaporating

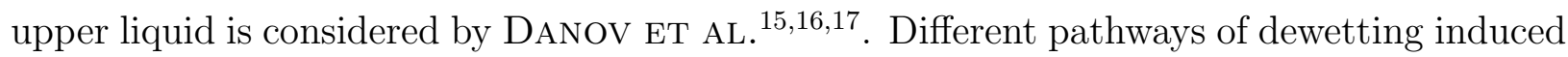

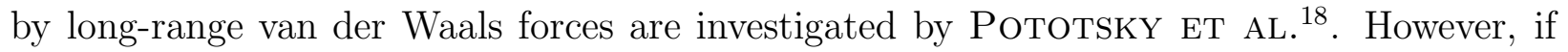
such a two-layer system is bounded below and above by rigid plates its behavior can be described by a single evolution equation for the liquid-liquid interface. This kind of system is treated in the present paper.

Although a general evolution equation was to our knowledge not yet given in the literature there exist a number of analyses for related systems. YiAntsios and HigGins ${ }^{19}$ investigate the Rayleigh-Taylor instability regarding an upper layer of infinite thickness. They use lubrication approximation for the lower liquid layer but not for the infinitely extended upper one. They find that to leading order the dynamics of the upper layer can be neglected if the viscosities of both liquids are of the same order of magnitude. In this way, they obtain an effective one-layer interface evolution equation.

Marangoni effects in two superposed fluid layers are experimentally studied by VANHoOK 
ET AL $\stackrel{20}{ }$. They investigate long-wave as well as short-wave thermocapillary instabilities. However, their theoretical analysis neglects velocities in the upper layer and uses a 'two-layer Biot number' to take into account the thermal properties as well as the thermal field in the upper layer. This generalization of the Biot number used in ${ }^{2}$ also leads to an effective onelayer equation. A similar theory is used by BURGESS ET AL. 21 to explain the stabilization of a Rayleigh-Taylor (RT) unstable oil-air system by heating form below. Linear investigations of short- and long-wave Marangoni instabilities in two superposed liquid films are presented by $\mathrm{Smith}^{22}$. Furthermore, Simanovski and NePOMNYASHCHY 23.24 consider a two-layer system with thermocapillary effects. They derive a weakly nonlinear interface equation in long-wave approximation taking into account the dynamics in both layers. Our linear results for the thermocapillary case can be directly compared to theirs. They show that the occurrence of thermocapillary instabilities is not only determined by the direction of the temperature gradient but also by the ratios of the viscosities and the layer thicknesses. In particular, they find that contrary to an one-layer system heating from above can act destabilizing. Moreover, TILley ET. $\mathrm{AL}^{25}$ investigate two superposed fluids in an inclined channel with gravity and Marangoni effects. Their weakly nonlinear analysis reveals a modified Kuramoto-Sivashinsky equation with broken reflectional symmetry.

Two superposed dielectric fluids between two parallel plates are an appropriate system to investigate pattern formation through electrohydrodynamic instabilities since a vertically applied electric field causes normal and tangential interface forces which depend strongly on the dielectric fluid properties. MAJUMDAR and O'NEILL ${ }^{26}$ propose an experimental method to quantify surface tension via the measured critical voltage for the onset of such an instability. MOHAMED ET AL.$^{27}$ investigate the destabilization of the interface using an Orr-Sommerfeld equation. Our linear results can be compared to theirs for quadratic 
velocity profiles since long-wave approximation allows for quadratic velocity profiles only. A detailed analysis of different electrical fluid properties like the creation of free charges at the interface, or the characterization of conducting and insulating fluids is given by MELCHER and $\mathrm{Smith}^{28}$. Investigations of a free thin liquid jet with an axial applied electric field are

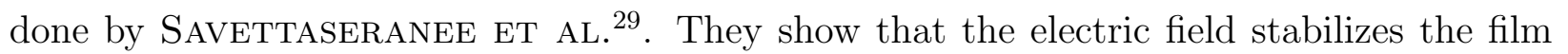
and can avoid rupture induced by attractive van der Waals forces. Experiments of LIN ET AL. ${ }^{30.31}$ using two layers of polymeric liquid exposed to a vertical electric field suggest that linear considerations do well capture the length scale found even in the long-time evolution. Our nonlinear calculations confirm the validity of the linear theories.

The present work focuses on two-layer films bounded by an upper and a lower rigid plate as sketched in Fig.1. In Section [1] we derive from the basic hydrodynamic equations the interface evolution equation in lubrication or long-wave approximation for general layer properties. Keeping the normal and tangential stresses at the interface in a general form, the resulting equation can be applied to the study of various body and interface forces. In the remaining paper we focus on gravity, thermocapillarity and electrostatic forces. This allows for the formulation of the problem in variational form using a Lyapunov functional. Since the free energy density is a function of the interface $h$ only, the long-time evolution can be predicted, i.e. whether holes, drops or maze structures are expected for $t \rightarrow \infty$. Further on we discuss the occurrence of metastable states. In Section [II] we perform linear and nonlinear analyses of the derived equation. First, we show that gravitation and thermocapillarity may act stabilizing as well as destabilizing depending on material and system parameters. Furthermore, we integrate the fully nonlinear evolution equation in three dimensions numerically and study the short-time as well as the long-time evolution. For the latter, different coarsening modes and the long-time scaling are discussed. We 
summarize our results in Section IV and point out possible applications, in particular the influence of electrostatic fields on dewetting. In the Appendix we discuss shortly the subtle occurrence of an additional mean flow field if the system is extended from 2D to 3D.

\section{GOVERNING EQUATIONS}

We consider a two-layer system bounded by a rigid upper and lower plate with a system thickness $d$ and a flat film interface with height $h_{0}$ (Fig.11). Instabilities lead to a time and space dependent interface profile $h(x, y, t)$.

\section{A. Evolution equation}

The required material parameters for incompressible fluids are the densities $\rho_{i}$ and the dynamic viscosities $\mu_{i}$. The subscripts $i=1$ and $i=2$ denote liquid 1 (lower layer) and liquid 2 (upper layer), respectively. In long-wave approximation the governing equations are found by a perturbation series in powers of the small parameter $\epsilon^{2}$. We write in two dimensions $(2 \mathrm{D})$

$$
\begin{aligned}
& u_{i}=u_{i_{0}}+\epsilon u_{i_{1}}+\epsilon^{2} u_{i_{2}}+\ldots \\
& w_{i}=w_{i_{0}}+\epsilon w_{i_{1}}+\epsilon^{2} w_{i_{2}}+\ldots \\
& P_{i}=P_{i_{0}}+\epsilon P_{i_{1}}+\epsilon^{2} P_{i_{2}}+\ldots
\end{aligned}
$$

where $u_{i}$ and $w_{i}$ stand for the $x$ - and $z$-components of the velocities, respectively. The small

parameter $\epsilon=\frac{2 \pi h_{0}}{\Lambda} \ll 1$ reflects the fact that the interface deflections are long scale, i.e. the mean film thickness $h_{0}$ is small compared to the typical lateral length scale $\Lambda$.

Taking the large difference in length scales into account, it is natural to scale the system lengths like $z=h_{0} z^{\prime}$ and $x=\frac{h_{0}}{\epsilon} x^{\prime}$, the velocities like $u_{i}=u_{0} u_{i}^{\prime}$ and $w_{i}=\epsilon u_{0} w_{i}^{\prime}$, the time 
like $t=\frac{h_{0}}{u_{0} \epsilon} t^{\prime}$, the pressures like $P_{i}=\frac{\mu_{1} u_{0}}{h_{0} \epsilon} P_{i}^{\prime}$, the body forces like $\Phi_{i}=\frac{\mu_{1} u_{0}}{h_{0} \epsilon} \Phi_{i}^{\prime}$, and the normal and tangential interface forces like $\Pi=\frac{\mu_{1} u_{0}}{h_{0}} \Pi^{\prime}$ and $\tau=\frac{\mu_{1} u_{0}}{h_{0}} \tau^{\prime}$. The primes denote the dimensionless variables. $u_{0}$ is a reference velocity of fluid 1 parallel to the substrate.

Starting from the two-dimensional incompressible Navier-Stokes equations and the continuity equations for both liquid layers we derive the dimensionless equations in zeroth order in $\epsilon$. After substituting Eqs. (11) in the governing equations and neglecting all terms of $O(\epsilon)$ or smaller, we drop the primes and the subscript zero and obtain for the scaled quantities in the lower layer, $0 \leq z \leq h(x, t)$

$$
\begin{aligned}
\partial_{z}^{2} u_{1} & =\partial_{x} \tilde{P}_{1} \\
\partial_{z} \tilde{P}_{1} & =0 \\
\partial_{x} u_{1}+\partial_{z} w_{1} & =0
\end{aligned}
$$

and in the upper layer, $h(x, t) \leq z \leq d$

$$
\begin{aligned}
\mu \partial_{z}^{2} u_{2} & =\partial_{x} \tilde{P}_{2} \\
\partial_{z} \tilde{P}_{2} & =0 \\
\partial_{x} u_{2}+\partial_{z} w_{2} & =0 .
\end{aligned}
$$

The variables

$$
\tilde{P}_{1}=P_{1}+\Phi_{1} \quad \text { and } \quad \tilde{P}_{2}=P_{2}+\Phi_{2}
$$

stand for reduced pressures which are the sum of the hydrostatic pressure $P_{i}$ and the potential of the conservative body force $\Phi_{i}$ (e.g. gravity force). The parameter $\mu=\mu_{2} / \mu_{1}$ represents the ratio of the dynamic viscosities. The boundary conditions at the lower and the upper boundaries read

$$
u_{1}=0, \quad w_{1}=0 \quad \text { at } \quad z=0
$$


and

$$
u_{2}=0, \quad w_{2}=0 \quad \text { at } \quad z=d .
$$

The resulting interface conditions in zeroth order at $z=h(x, t)$ are

$$
\begin{aligned}
\tilde{P}_{1}-\tilde{P}_{2} & =\mathcal{N}+\Phi \\
\partial_{z} u_{1}-\mu \partial_{z} u_{2} & =\mathcal{T} \\
\partial_{t} h+u_{1} \partial_{x} h & =w_{1} \\
\partial_{t} h+u_{2} \partial_{x} h & =w_{2} \\
u_{1} & =u_{2} \\
w_{1} & =w_{2}
\end{aligned}
$$

In the remainder of the paper we use the abbreviations $\mathcal{N}$ for the normal forces Eq. (5a), $\mathcal{T}$ for the tangential forces at the interface Eq. (5b) and $\Phi=\Phi_{1}-\Phi_{2}$ for the body force potential. We mention that the occurrence of the body force potential $\Phi$ in Eq. (15a) is caused by using reduced pressures Eq. (3).

Because $\tilde{P}_{1}$ and $\tilde{P}_{2}$ do not depend on $z$ (Eqs. (2b) and (20) ) one can integrate Eqs. (2a (2d) in $z$. With the boundary conditions Eq. (44) and the interface conditions Eqs. (5b), (5e) the $x$-components of the velocities take the explicit form

$$
\begin{aligned}
& u_{1}(x, z, t)=\frac{1}{2} \partial_{x} \tilde{P}_{1} z^{2}+\left(-h \partial_{x} \mathcal{N}-h \partial_{x} \Phi+B+\mathcal{T}\right) z \\
& u_{2}(x, z, t)=\frac{1}{2 \mu}\left(\partial_{x} \tilde{P}_{1}-\partial_{x} \mathcal{N}-\partial_{x} \Phi\right)\left(z^{2}-d^{2}\right)+\left(\frac{1}{\mu} B\right)(z-d) \\
& \text { with } B=\frac{1}{2} \frac{-\left(\partial_{x} \tilde{P}_{1}-2 \partial_{x} \mathcal{N}-2 \partial_{x} \Phi\right) \mu h^{2}+\left(\partial_{x} \tilde{P}_{1}-\partial_{x} \mathcal{N}-\partial_{x} \Phi\right)\left(h^{2}-d^{2}\right)-2 \mu \mathcal{T} h}{(\mu-1) h+d}
\end{aligned}
$$

where we used Eq. (5a) to express $\partial_{x} \tilde{P}_{2}$ as a function of $\partial_{x} \tilde{P}_{1}$.

Next, we derive the evolution equation for the interface profile $h(x, t)$ and an explicit formula for the pressure $\partial_{x} \tilde{P}_{1}$. To do so the continuity equation Eq. (2C) is integrated in $z$-direction. 
With the interface condition Eq. (5c) and the chain rule we find

$$
\partial_{t} h+\partial_{x} \int_{0}^{h(x, t)} u_{1}(x, z, t) d z=0 .
$$

A similar evolution equation for $h(x, t)$ is also derived for the upper layer using Eqs. (2f) and (5d). Then Eq. (5f) yields the identity

$$
\partial_{x}\left(\int_{0}^{h(x, t)} u_{1}(x, z, t) d z+\int_{h(x, t)}^{d} u_{2}(x, z, t) d z\right)=0 .
$$

To obtain $\tilde{P}_{1}$, Eq. (8) is integrated in $x$ setting the integration constant to zero without loss of generality. This can be done if there is no additional lateral driving force as, for instance, in an inclined system. The resulting equation is solved using Eq. (44). The resulting pressure gradient is

$$
\partial_{x} \tilde{P}_{1}=F_{1}(h) \partial_{x}(\mathcal{N}+\Phi)+F_{2}(h) \mathcal{T}
$$

with

$$
\begin{aligned}
F_{1}(h) & =\frac{1}{D}(d-h)^{2}\left(h \mu(4 d-h)+(d-h)^{2}\right) \\
F_{2}(h) & =\frac{6 \mu d h}{D}(d-h) \\
D & =(d-h)^{4}+h \mu\left(h^{3}(\mu-2)+4 d h^{2}-6 d^{2} h+4 d^{3}\right) .
\end{aligned}
$$

The evolution equation Eq. (71) can be written (with Eq. (6a)) as

$$
\partial_{t} h=\partial_{x}\left[Q_{1}(h) \partial_{x}(\mathcal{N}+\Phi)+Q_{2}(h) \mathcal{T}\right]
$$

Using the same procedure we derive a similar equation for three dimensions

$$
\partial_{t} h=\nabla \cdot\left[Q_{1}(h) \nabla(\mathcal{N}+\Phi)+Q_{2}(h) \overrightarrow{\mathcal{T}}\right]
$$

where $\nabla=\left(\partial_{x}, \partial_{y}\right)$. We note that in $3 \mathrm{D}$ an additional field occurs which can not be expressed as a function of $h$ analytically. The importance and the equation of this mean flow field are discussed in the appendix. However, we neglect the mean flow in the following. 
The mobilities are

$$
\begin{aligned}
& Q_{1}(h)=\frac{h^{3}(d-h)^{3}}{3 D}[d+h(\mu-1)] \\
& Q_{2}(h)=\frac{h^{2}(d-h)^{2}}{2 D}\left[h^{2}(\mu-1)-d(d-2 h)\right] .
\end{aligned}
$$

The mobility $Q_{1}(h)$ is positive for all values $\mu>0$ and $d>0$. It vanishes for $h=0$ and $h=d$. However, $Q_{2}(h)$ always changes its sign at

$$
h_{c}=\frac{d}{\sqrt{\mu}+1} .
$$

The mobility $Q_{2}(h)$ does only exist in a system with shear-stress. In Fig.2 the mobilities are plotted for the parameters of Tab.I with $d=1.3$. There the change of sign of $Q_{2}(h)$ occurs at $h_{c} \approx 0.91$.

In an one-layer system the viscosity of the upper gas layer is neglected. Therefore, taking the limit $\mu \rightarrow 0$ of the mobilities Eqs. (13) give the correct one-layer mobilities

$$
Q_{1}(h)_{\lim }=\frac{1}{3} h^{3}, \quad \text { and } \quad Q_{2}(h)_{\lim }=-\frac{1}{2} h^{2},
$$

and the evolution equation Eq. (12) takes the well known form of the thin film equation for a single layer ${ }^{2}$

$$
\partial_{t} h=-\nabla\left[-\frac{1}{3} h^{3} \nabla \tilde{P}_{1 \lim }+\frac{1}{2} h^{2} \mathcal{T}_{\lim }\right]
$$

The limit of the pressure is

$$
\tilde{P}_{1 \lim }=P_{1}+\Phi_{1}
$$

since $\nabla \tilde{P}_{2}=0$ (see Eq. (2d $)$ ) and $\Phi_{2}$ can be neglected as can be seen, for example, for gravity forces where $\Phi_{i} \propto \rho_{i}$ and $\rho_{2} \ll \rho_{1}$ leads directly to $\Phi_{1} \gg \Phi_{2}$. The same limit is reached by increasing the system thickness $d \rightarrow \infty$. 


\section{B. Specific effects}

\section{Gravitation and surface tension}

Incorporation of gravitation and surface tension (capillarity) provides the body force potential and the normal interface force

$$
\begin{aligned}
\Phi_{G} & =(1-\rho) G h \\
\text { and } \mathcal{N} & =-C^{-1} \nabla^{2} h,
\end{aligned}
$$

respectively. Thereby, $G=\left(\rho_{1} g h_{0}^{2}\right) /\left(\mu_{1} u_{0}\right)$ is the gravity number, $\rho=\rho_{2} / \rho_{1}$ denotes the ratio of densities, $C^{-1}=\epsilon^{3} \sigma /\left(\mu_{1} u_{0}\right)$ denotes the capillary number and $\sigma$ is the dimensional liquid-liquid surface tension.

\section{Thermocapillarity}

The equations for the non-dimensional temperatures $\Theta_{i}$ (energy equations) read in zeroth order in $\epsilon$

$$
\begin{aligned}
& \partial_{z}^{2} \Theta_{1}=0 \\
& \partial_{z}^{2} \Theta_{2}=0 .
\end{aligned}
$$

The non-dimensional temperature is defined by

$$
\Theta_{i}=\frac{T_{i}-T_{u}}{T_{l}-T_{u}}
$$

where $T_{u}$ and $T_{l}$ refer to the temperature at the upper and lower plate, respectively. The boundary conditions at the lower and upper rigid plate are

$$
\begin{aligned}
& \Theta_{1}=1 \quad \text { at } \quad z=0 \\
& \text { and } \Theta_{2}=0 \quad \text { at } z=d
\end{aligned}
$$


and the interface conditions at $z=h(x, t)$ read

$$
\begin{gathered}
\Theta_{1}=\Theta_{2} \\
\partial_{z} \Theta_{1}=\lambda \partial_{z} \Theta_{2},
\end{gathered}
$$

where $\lambda=\lambda_{2} / \lambda_{1}$ is the ratio of the thermal conductivities. Eqs. (19) together with Eqs. (21) and (22) give the temperature fields

$$
\begin{aligned}
\Theta_{1}(z) & =\frac{\lambda(h-z)+(d-h)}{(\lambda-1) h+d} \\
\text { and } \quad \Theta_{2}(z) & =\frac{d-z}{(\lambda-1) h+d} .
\end{aligned}
$$

Assuming an arbitrary dependence of the surface tension $\sigma$ on temperature, the tangential interface condition Eq. (5b) has the form $\mathcal{T}=\nabla \Sigma$ where $\Sigma=\epsilon \sigma /\left(\mu_{1} u_{0}\right)$ is the dimensionless surface tension. Evaluation of $\nabla \Sigma$ at the position $z=h(x, y, t)$ gives $\nabla \Sigma=\partial_{\Theta} \Sigma \cdot \partial_{h} \Theta(h) \cdot \nabla h$. If the surface tension depends linearly on temperature one gets

$$
\mathcal{T}=M \frac{\lambda d}{[(\lambda-1) h+d]^{2}} \nabla h
$$

where $M=\left(-\partial_{T} \sigma \Delta T \epsilon\right) /\left(\mu_{1} u_{0}\right)$ is the Marangoni number, $\partial_{T} \sigma$ is the change of surface tension with temperature and $\Delta T=T_{l}-T_{u}$ is the applied temperature difference. If the system is heated from below, $M$ is positive for most fluids (normal thermocapillary effect). To derive the one-layer limit of the thermocapillary force $\mathcal{T}_{\lim }$ one replaces $\lambda$ with a (conductive) Biot number $\lambda=B i(d-1)$ in Eq. (24) and takes the limit $d \rightarrow \infty$. Considering the gas layer as a semi-infinite layer, we get the usual one-layer expression²

$$
\mathcal{T}_{\lim }=M \frac{B i}{(B i h+1)^{2}} \nabla h
$$

where $B i \ll 1$. We note that the 'two-layer Biot number', $B i_{2}$, introduced by VANHook ET AL.$^{20}$ can be obtained by replacing $\lambda=\frac{1-B i_{2}(d-1)}{1+B i_{2}}$ in Eq. (24) and taking the limit $d \rightarrow 1$. 
This limit is valid since all dependencies on $d$ are considered to be in $B i_{2}$ and therefore the neglect of the upper layer leads to $d \rightarrow 1$.

\section{Disjoining pressure}

To avoid film rupture at the two bounding plates we incorporate disjoining pressures in the body force potential to model repelling stabilizing van der Waals forces ${ }^{10,32}$

$$
\begin{aligned}
\Phi_{D_{1}} & =-\left.\frac{H_{1}}{z^{3}}\right|_{z=h} \\
\Phi_{D_{2}} & =-\left.\frac{H_{2}}{(d-z)^{3}}\right|_{z=h} .
\end{aligned}
$$

The parameters $H_{1}$ and $H_{2}$ are Hamaker constants representing the interaction of the lower plate with liquid 2 through liquid 1 and of the upper plate with liquid 1 through liquid 2 , respectively ${ }^{13}$. Thereby, we neglect a part of the forces between the lower (upper) fluid and the upper (lower) substrate resulting from the finite thickness of the respective layer. The Hamaker constants determine the macroscopic contact angles. Since large macroscopic contact angles violate the used long-wave approximation we use Hamaker constants which provide a small contact angle. These corresponds to fluids which partially wet the substrates. Note, that for a very small distance between the plates, i.e. if both layers are ultrathin with thicknesses below $100 \mathrm{~nm}$ the disjoining pressure has the most important influence and all

forces have to be included. Such systems are not the scope of the present work, but see $\frac{18}{}$ for a related system.

\section{Electrostatic field}

An electric field applied in $z$-direction is another way to cause structure formation. Consider two dielectric fluids with permittivities $\varepsilon_{1}$ and $\varepsilon_{2}$, respectively. The upper and lower plates 
serve as electrodes and a voltage $U$ is applied. The vertical components of the electric fields in fluid 1 and 2 read then in zeroth order lubrication approximation

$$
\begin{aligned}
E_{1} & =\frac{\varepsilon_{2} U}{\varepsilon_{2} h+\varepsilon_{1}(d-h)} \\
\text { and } \quad E_{2} & =\frac{\varepsilon_{1} U}{\varepsilon_{2} h+\varepsilon_{1}(d-h)} .
\end{aligned}
$$

Horizontal components can be neglected at this order. Using the electrohydrodynamic stress tensor $^{33}$ for $\rho_{i}=$ const., provides the effective electrostatic pressure at the interface by projecting the stress tensor two times on the normal vector

$$
p_{e l}=\frac{1}{2} \varepsilon_{0}\left(\varepsilon_{2}-\varepsilon_{1}\right) E_{1} E_{2}
$$

Scaling the voltage like $U=U^{\prime} \sqrt{\mu_{1} u_{0} h_{0} / \varepsilon_{0} \varepsilon_{1} \epsilon}$ and dropping the primes, leads in zeroth order lubrication approximation to

$$
\mathcal{N}_{e l}=\frac{1}{2} \frac{\varepsilon(\varepsilon-1) U^{2}}{(\varepsilon h+(d-h))^{2}},
$$

where $\varepsilon_{0}$ denotes the permittivity of vacuum and $\varepsilon=\varepsilon_{2} / \varepsilon_{1}$ is the ratio of permittivities. In zeroth order in $\epsilon$ shear stresses $\mathcal{T}_{\text {el }}$ are not present.

\section{Energy}

As for one-layer systems ${ }^{8}$ also here it is possible to express the r.h.s. of the evolution equation Eq. (12) in variational form

$$
\partial_{t} h=\nabla \cdot\left[Q_{1}(h) \nabla \frac{\delta E}{\delta h}\right]
$$

corresponding to the evolution equation of a conserved order parameter field in a relaxational

situation. Incorporating the above mentioned effects the energy $E$ that corresponds to a Lyapunov functional can be written as

$$
E=\iint d x d y\left[\frac{1}{2} C^{-1}(\nabla h)^{2}+V(h)\right]
$$


with

$$
V(h)=\frac{1}{2}(1-\rho) G h^{2}+\frac{H_{1}}{2} h^{-2}+\frac{H_{2}}{2}(d-h)^{-2}+E_{t h}(h)+E_{e l}(h),
$$

and

$$
\begin{aligned}
E_{t h}(h)= & \frac{3 M}{2 d \lambda(\mu-\lambda)^{2}}\left[-\lambda^{2}(\mu-\lambda)^{2} h \ln (h)+(\mu-\lambda)^{2}(d-h) \ln (d-h)\right. \\
& +\lambda^{2}(\mu-1)(h(\mu-1)+d) \ln (h(\mu-1)+d) \\
& \left.+\left(\lambda^{4} h-2 \lambda^{3} \mu h+\lambda^{2} \mu(2 h-d)+2 \lambda \mu(d-h)-\mu^{2}(d-h)\right) \ln (h(\lambda-1)+d)\right]
\end{aligned}
$$

for thermocapillarity and

$$
E_{e l}(h)=-\frac{1}{2} \frac{\varepsilon U^{2}}{(\varepsilon-1) h+d}
$$

for electrostatic fields.

It can easily be shown ${ }^{2.34}$ that the Lyapunov functional $E$ is monotonously decreasing in time $\left(\frac{d}{d t} E \leq 0\right)$, if the mobility $Q_{1}(h)>0$ which is always fulfilled. Note, that the free energy density for thermocapillarity $E_{t h}$ is a function of the ratios of viscosities $\mu$, thermal conductivities $\lambda$ and layer thicknesses $d$. The dependence of $E_{t h}$ on the viscosities, i.e. on a dynamical aspect of the system, does not occur in a one-layer system.

\section{RESULTS AND DISCUSSION}

\section{A. Material parameters}

For our numerical investigations we focus on one specific two-layer system to allow for a direct comparison to experiments. We chose an oil-oil system used in Ref. $\frac{35}{}$, namely silicon oil $5 \mathrm{cS}$ and HT70. The parameter values are given in Tab.I. Note, that the given permittivities are only a rough estimate. 


\section{B. Linear stability}

To solve the linear problem the normal mode ansatz

$$
h(x, y, t)=h_{k} \exp \left(i k_{x} x+i k_{y} y+\chi t\right)
$$

is used in Eq. (12). Linearization provides the growth rate $\chi$

$$
\begin{aligned}
\chi & =-\frac{Q_{1}(1)}{C} k^{2}\left(k^{2}-k_{c}^{2}\right) \\
\text { with } \quad k_{c}^{2} & =C\left[(\rho-1) G+\frac{\varepsilon(\varepsilon-1)^{2} U^{2}}{(\varepsilon-1+d)^{3}}-3 H_{1}-\frac{3 H_{2}}{(d-1)^{4}}-\frac{Q_{2}(1)}{Q_{1}(1)} \frac{\lambda d M}{(\lambda-1+d)^{2}}\right]
\end{aligned}
$$

where $k_{c}$ is the cut-off or critical wavenumber and $k^{2}=k_{x}^{2}+k_{y}^{2}$. The system is unstable for positive growth rates $\chi>0$, i.e. for $k<k_{c}$. Onset of the instability occurs with infinite wave length when $k_{c}=0$.

\section{Gravitation, surface tension and thermocapillary effects}

First, we study the situation without electric field, i.e. $U=0$. For $H_{1}, H_{2} \ll 1$ the linear stability is determined by $\rho$ and $M$ only. In the isothermal case $(M=0)$ the system is unstable for $\rho>1$, i.e. the system is gravitationally or Rayleigh-Taylor (RT) unstable. In the heated case, Eq. (36) provides the critical Marangoni number

$$
M_{c}=-\frac{2}{3} \frac{(\lambda-1+d)^{2}(d-1)(\mu+d-1)}{\lambda d\left(\mu-(d-1)^{2}\right)}\left((1-\rho) G+3 H_{1}+\frac{3 H_{2}}{(d-1)^{4}}\right) .
$$

We note that the critical Marangoni number correspond to the one found by $\mathrm{SmITH}^{22}$ for thin layers of viscous liquids. Inspection of Eq. (36) shows that the sign of the thermocapillarity term does not only depend on the sign of the Marangoni number but also on the sign of the mobility $Q_{2}(1)$. This implies that the sign of $Q_{2}(1)$ determines whether $M$ must be larger or smaller than $M_{c}$ to get an instability. Denoting the zero crossing of $Q_{2}(h)$ by $h_{c}$ 
(see Eq. (14)), one finds that for $h_{c}>1$ the Marangoni number $M$ has to be increased over $M_{c}$ for the system to become unstable, whereas for $h_{c}<1$ it has to be decreased below $M_{c}$. The destabilizing direction of heating is determined by substituting $h_{c}=1$ in Eq. (14). Instability results if

$$
\begin{aligned}
& \mu<(d-1)^{2} \quad \text { for } \quad M>M_{c} \\
& \text { and if } \quad \mu>(d-1)^{2} \quad \text { for } \quad M<M_{c} \text {. }
\end{aligned}
$$

The critical system thickness (critical viscosity ratio, respectively) reads then

$$
d_{c}=\sqrt{\mu}+1 \quad\left(\mu_{c}=(d-1)^{2}\right)
$$

as already found in Ref. 24 . The dependence of the growth rate on the wavenumber is shown in Fig. 3 for three different situations at a system thickness $d=1.3$, parameters from the first column in Tab.【 and $H_{1}=H_{2}=0$. Because $\rho=\rho_{2} / \rho_{1}>1$ without heating the system is Rayleigh-Taylor unstable (solid line). Since the system thickness is smaller than the critical one $d_{c}=1.43$ (Eq. (40) $)$, heating from below with $M>M_{c}=0.89$ damps the RT instability (dashed line). As indicated in Eq. (39b) heating from above amplifies the Rayleigh-Taylor instability (dotted line). The stabilization mechanism for $M>M_{c}$ is directly correlated with the sign of the mobility $Q_{2}(h)$. The zero crossing of $Q_{2}(h)$ is at $h_{c} \approx 0.91$ for the chosen material parameters. Therefore, the mobility $Q_{2}$ is positive in the linear regime $(h \approx 1)$ and heating from below $(M>0)$ acts stabilizing.

The stability diagrams in Fig. 4 show the critical Marangoni number $M_{c}$ (Eq. (38) ) in dependence of the ratio of viscosities $\mu$ for $d=1.3$ and $\lambda$ from the first column in Tab.【 $\left(H_{1}=H_{2}=0\right)$. The left (right) panel represents a system that is Rayleigh-Taylor unstable (stable) for $M=0$. At the critical viscosity $\mu_{c}=0.09$ the critical Marangoni number $M_{c}$ changes its algebraic sign in both cases. Obviously, thermocapillarity dominates for strong 
heating (large $|M|$ ), i.e. RT is negligible.

To understand the mechanism of the stabilization of a RT instability for $M>M_{c}$ and $\mu>\mu_{c}$, we consider a small deformation of the interface in negative $z$-direction as sketched in Fig. 5 . First, we discuss the isothermal case $(M=0)$ where for $\rho>1$ the system evolves due to its Rayleigh-Taylor instability. The viscous time scales of the two layers are responsible for the direction of the fluid velocity in the vicinity of the deformation minimum. For $d>d_{c}$ the viscous time scale of the lower layer $\tau_{1} \propto h_{0}^{2} / \mu_{1}$ is faster than the viscous time scale $\tau_{2} \propto\left(d-h_{0}\right)^{2} / \mu_{2}$ of the upper one. Therefore, the lower layer is the driving layer and velocities are directed away from the deformation minimum (solid arrows in Fig.[5](a)). For $d<d_{c}$ the velocities are directed towards the deformation (solid arrows in Fig.國(b)).

When heated from below $(M>0)$ the temperature is highest at the deformation minimum. The accompanying surface tension gradient causes a flow away from the minimum (dashed arrows in Fig. (5) . This leads to an amplification of the perturbation for $d>d_{c}$, because thermocapillarity acts in the same direction as the Rayleigh-Taylor mechanism (Fig.5(a)). For $d<d_{c}$ thermocapillary forces act in the same direction as before, but the driving of the upper layer leads to flow towards the deformation minimum (Fig.[5(b)). Therefore thermocapillarity damps out the Rayleigh-Taylor instability.

Finally, we display in Fig. [6] the critical Marangoni number $M_{c}$ in its dependence on the system thickness $d$ as calculated from Eq. (38). To avoid a destabilizing influence of a Rayleigh-Taylor instability we take the parameters from the second column in Tab.】 i.e. we interchange the two liquids. For $d>d_{c} \approx 3.34$ a minimum in $M_{c}$ is observed. It reflects the antagonistic influences of the system thickness and the temperature gradient in the lower layer (driving layer). For large $d$ the temperature gradient in the lower layer tends to zero (from Eqs. (21) and (23)), implying a large critical Marangoni number. Decreasing the 
system thickness leads to decreasing $M_{c}$. When decreasing $d$ further the mobility $Q_{2}(h=1)$ tends to zero. Hence, for $d \rightarrow d_{c}$ one again finds an increasing $M_{c}$.

For system thicknesses $d<d_{c}$ one observes a monotonously decreasing $\left|M_{c}\right|$ for $d \rightarrow 1$. Including disjoining pressures as repelling forces changes the behavior for very small thickness of the upper layer $d-1$ qualitatively, but has no influence otherwise (dashed line). Specifi-

cally, for $d<d_{c}$ the Hamaker constant of the upper layer $H_{2}$ (representing the interaction of the upper substrate with liquid 1 through liquid 2) forces an extremum of $M_{c}$. Decreasing the thickness of the upper layer towards $d \approx 1$ the stabilizing Van der Waals interaction finally dominates allowing to consider systems with very small $d-1$ as stable. The Hamaker constant $H_{1}$ (representing the interaction of the lower substrate with liquid 2 through liquid 1) causes only a slight shift of $M_{c}$. It does not change the extremum in this region.

\section{Electrostatic field}

We conclude the discussion of the linear stability by regarding the influence of a vertical electrical field only. Neglecting thermocapillarity $(M=0)$ and gravitation $(G=0)$ yields for $H_{1}, H_{2} \ll 1$ the critical voltage from Eq. (36)

$$
U_{c}=\sqrt{3\left(H_{1}+\frac{H_{2}}{(d-1)^{4}}\right) \frac{(\varepsilon-1+d)^{3}}{\varepsilon(\varepsilon-1)^{2}}} .
$$

Note, that the direction of the applied voltage $( \pm z)$ has no influence on the stability. Using parameters of the second column in Tab. [ with $H_{1}=H_{2}=0.01$ and $d=4$ we find the critical voltage $U_{c} \approx 4.5$. A voltage of $U=30$ provides the wavenumber $k_{\max } \approx 0.18$ for the maximal growth rate $\chi$. We use these parameters to study the time evolution with the fully nonlinear equation below in Section 


\section{Implications of the variational formulation}

The variational formulation of the evolution equation (Eq. (30) ) provides an energy or Lyapunov functional based on a gradient energy and a free energy density $V(h)($ Eq. (32) $)$. The latter can be used in a Maxwell construction that allows for the prediction of the character of the resulting structure in the long-time evolution ${ }^{10}$ as well as the study of metastable states ${ }^{11}$.

\section{Maxwell construction}

First, we want to determine whether holes or drops are formed in the long-time evolution. Since a Lyapunov functional Eq. (31) exists, the final equilibrium thickness profile corresponds to its global minimum. The mean height $h_{0}$ is a conserved quantity, i.e. an increase of the interface height in any region is accompanied by a decrease somewhere else. When minimizing the energy functional, this constraint has to be taken into account by a Lagrange multiplier, $\lambda_{L}$, namely by supplementing the free energy density $V(h)$ by the term $\lambda_{L} h$. Assuming a very large system in a late stage of coarsening the gradient term of the energy functional can be neglected and the local free energy suffices to derive the long-time behavior. First, consider a $V(h)$ possessing one minimum and a monotonously increasing slope, i.e. a $V(h)$ with positive second derivative everywhere. Then, deforming the interface increases the local part of the free energy functional (which is further increased due to the gradient term) since the energy loss is due to mass conservation accompanied by a larger energy gain. Therefore, for such a $V(h)$ the (energetically minimized) final solution is a flat interface.

Contrary to this, a $V(h)$ with two minima may allow to minimize the local free energy (and may even overcome the energy gain due to the gradient term) by deforming the interface 
since the free energy may decrease for both $h>h_{0}$ and $h<h_{0}$. In this case the flat interface is linearly unstable and the system will realize two film thicknesses $\left(h_{1}<h_{0}=1<h_{2}\right)$. In analogy to spinodal decomposition the two film thicknesses can be seen as two different phases, and the evolution of the film thickness profile corresponds to a phase separation $\underline{36}$. Mathematically formulated, the phase separation occurs if it is possible to find a double tangent, where the curve $V(h)$ lies everywhere above this tangent. The slope of the tangent corresponds to the Lagrange multiplier $\lambda_{L}$, and the points where it touches the curve $V(h)$ give the two equilibrium values of $h$. In the present case the existence of the double tangent is assured by the stabilizing disjoining pressures. Without the latter the equilibrium film thicknesses may be found outside the gap between the two substrate indicating finite 'true' contact angles. The double tangent condition is equivalent to a Maxwell construction in the $\left[-d_{h} V(h)\right]-[h]$ space.

Resulting from mass conservation, the ratio of the surface areas $S=S_{1} / S_{2}$ of the two equilibrium film thicknesses $\left(h_{1}<h_{0}\right.$ and $\left.h_{2}>h_{0}\right)$ defines the solution morphology. In accordance with observed structures in thin films we call $S<1, S>1$ and $S=1$ hole, drop and maze solutions, respectively. However, for systems with $S$ close to 1 but $S<1$ $(S>1)$ the solution reveals its visible hole (drop) character not until the final stationary state. Note, that we use the expression 'hole' ('drop') for a hole (drop) in (of) the lower fluid. Obviously a hole (drop) in (of) the lower fluid corresponds to a drop (hole) of (in) the upper fluid.

We focus on the system thickness $d$ as control parameter for the phase separation since $d$ can be controlled easily in experiments. Fig. [7(a) shows the plot of $-d_{h} V(h)$ for different system thicknesses $d$. The Maxwell point $h_{M}$ (via a Maxwell construction) provides then a criterion for holes or drops. If $h_{M}>1(S>1)$ drops are preferred (dashed line), in the 
other case $(S<1)$ holes are expected (solid line). For $d=2$ the transition from holes to drops takes place (dotted line). We mention, that this Maxwell point $h_{M_{2}}$ does not coincide with the critical system thickness $d_{c}$.

\section{Metastability}

As stated in the linear investigation above in Section IIIB 1, the Rayleigh-Taylor (RT) instability can be stabilized by heating from below (for $d<d_{c}$ ). This is also confirmed by a fully nonlinear integration in time (not shown). Nevertheless, a RT unstable system stabilized by thermocapillarity can be metastable, i.e. it may be nonlinearly unstable to (large) finite perturbations. This metastability can also be studied using a Maxwell construction as shown in Fig.7(b). Under isothermal conditions (dashed line) the system is RT unstable indicated in Fig.[7(b) by the fact that the vertical line at $h=1$ crosses the dashed curve in-between the two extrema. When heating from below with $M=10$ (solid line) the system is linearly stable. However, the Maxwell plot has still two extrema. Since the mean system thickness $\left(h_{0}=1\right)$ is situated to the right of the maximum and the local free energy $(V(h=1))$ is larger then the free energy of the Maxwell point $\left(V\left(h_{m}\right)\right)$ the system is metastable.

Fig. [8] shows the critical Marangoni number $M_{c}$ in dependence of the system thickness $d$. For $d<d_{c}\left(d>d_{c}\right)$ heating from below (above) with $M>M_{c}\left(M<M_{c}\right)$ stabilizes the system. However, a Maxwell construction shows a metastable state for all the displayed Marangoni numbers $(|M|<60)$. This metastability was also found in experiments with oil-air layers ${ }^{21}$. A qualitative understanding of the metastability is given by the zero crossing $h_{c}$ of the mobility $Q_{2}$ (Eq. (14) $)$. For perturbations larger than $\left|1-h_{c}\right|$ the interface is destabilized since both gravity and thermocapillarity destabilize the system.

Fig.9 shows a snapshot from a two-dimensional numerical run for a RT instability without 
thermocapillarity (dashed line). Heating from below $(M=10)$ stabilizes the system and leads to a flat stable interface for small perturbations (not shown). However, starting with a finite perturbation in the vicinity of $x=50$ leads to a state with a local strong modulation (solid line).

\section{Long time evolution}

Three-dimensional numerical integrations of the nonlinear equation Eq. (12) are done with an ADI method (Alternating Discretization Integration). In the first half time step the linear part is integrated implicitly in $x$-direction, in the second half time step in $y$-direction. The nonlinear part is calculated explicitly. We use periodic boundary conditions in $x$ and $y$ and initially disturb the flat interface with small random fluctuations $\eta(x, y, t)$. Thereby the average height is conserved $\left(\int 1+\eta(x, y, t) d x d y=h_{0}=1\right)$. Further on, we distinguish between short-time evolution and long-time evolution. Roughly speaking linear effects determine the dominant length scales of the short-time evolution. Nonlinear effects dominate the long-time evolution that is characterized by coarsening processes.

\section{Rayleigh-Taylor instability}

In the isothermal case without electric fields, gravity is the only possibly destabilizing influence. The long-time evolution of a system with $d=3>d_{c}, G=5$ and material parameters from the first column in Tab. [1 is shown in Fig.10. Initially small disturbances of the flat interface evolve into a drop structure $(t \approx 1000)$. For larger times small drops vanish due to coarsening and finally the system settles at the global energetic minimum corresponding to one large drop (not shown). Fig.11 gives the evolution for $d=1.3<d_{c}$ and $G=20$. Here, 
the short-time evolution results in a hole pattern $(t<200)$. Subsequently, the long-time coarsening towards structures of larger extension sets in $(t=1000)$ and finally ends with one large hole $\left(t>2 \times 10^{5}\right.$, not shown).

The use of $G=20$ for $d=1.3$ and not $G=5$ as for $d=3$ assures a smooth growth in the short-time evolution. By 'smooth' we mean a gradual growth of all holes until they have rather large amplitudes. Using instead $G=5$ for $d=1.3$ results in a rapid non-smooth hole evolution in-between the short-time and long-time domain, i.e. the structure is determined by the linear wavelength at the very beginning of the evolution only. As soon as nonlinear terms become important $(|\eta(x, y . t)| \ll 1$ is violated) only part of the linearly developed structure evolves. Here, this rapid hole evolution is caused by the stabilizing mechanism of the disjoining pressure at the upper plate (Hamaker constant $H_{2}=0.01$ ) which can no longer be neglected even for small perturbations of the flat interface. This affects both the linear and the nonlinear evolution of the interface. Namely, it suppresses interface evolutions for $h>1$ and therefore causes a rapid evolution for $h<1$. We note again, that here the rapid hole evolution is caused by disjoining pressures, whereas the mobility $Q_{1}(h)$ has no effect.

Fig.12 displays the time evolution for $d=2, G=5$, and parameters from the first column of Tab.【. For these parameters, neither drops nor holes are energetically preferred. This leads to a clearly visible maze or labyrinth structure which also shows the typical coarsening dynamics at long times. All long-time solutions shown (drops for $d=3$, holes for $d=1.3$ and maze structures for $d=2$ ) correspond to the predictions of the Maxwell construction in Fig. [7] 
To quantify the coarsening behavior we calculate at each timestep the mean wave number

$$
\langle k\rangle(t)=\frac{\int_{k_{x}} \int_{k_{y}} d k_{x} d k_{y} \sqrt{\vec{k}^{2}} \tilde{h}^{2}\left(k_{x}, k_{y}, t\right)}{\int_{k_{x}} \int_{k_{y}} d k_{x} d k_{y} \tilde{h}^{2}\left(k_{x}, k_{y}, t\right)}
$$

where $\tilde{h}\left(k_{x}, k_{y}, t\right)$ denotes the Fourier transform of $h(x, y, t)$. We mention, that the wavevectors are distributed on a small annulus. Therefore the approximation $\langle k\rangle^{2} \approx\left\langle k^{2}\right\rangle$ holds and the mean wave number $\langle k\rangle$ can also be taken as a qualitative measure of the mean curvature of $h(x, y, t)$. Fig. [13 shows the dependence of $\langle k\rangle$ and of the corresponding energy (Eq. (31)) on time for the three numerical evolutions discussed above. The energy decreases always monotonously in time as expected. The mean wave number $\langle k\rangle$ shows two local extrema at $t_{\min }$ and $t_{\max }$, respectively, with $t_{\min }<t_{\max }$. In the region between the two extrema the amplitude of the interface deflections outgrows the linear regime. Further on, the averaging in Eq. (42) allows to interpret the strength of the maximum as a measurement for global amplitude growth. The absence of a local maximum indicates that all linearly evolved drops or holes evolve globally and uniformly towards larger amplitudes, whereas a strong peak indicates a nonlinear evolution of a few linearly evolved drops (holes) only.

The mean wave number at the local minimum corresponds to the wave number of the maximal growth rate from the linear investigation (thin horizontal lines). Hence the evolution for $t<t_{\min }$ is determined by linear terms, i.e. the wavenumber with the maximal linear growth rate emerges in the system. Therefore, the region around the two extrema can be considered as the frontier between short-time and long-time evolution. In the long-time evolution $\left(t>t_{\max }\right)$ nonlinear effects dominate and a scaling law $\underline{10}$

$$
\langle k\rangle=c \cdot t^{-\beta}
$$

can be extracted which reflects the coarsening of the system for long times. To determine 
a 'true' scaling exponent a statistically significant average of many numercial runs with different initial conditions is necessary. Due to the strongly time-consuming character of the necessary computer calculations only a few runs were used to determine the respective tendencies of scaling exponents presented here. However, in the following we call them shortly 'scaling exponents'.

We find a nearly identical scaling exponent of $\beta \approx 0.14$ for drops $\left(d=3\right.$, solid line, $t_{\max } \approx$ $150)$, holes $\left(d=1.3\right.$, dashed line, $\left.t_{\max } \approx 210\right)$ and maze structures $(d=2$, dotted line, $\left.t_{\max } \approx 320\right)$. Neither the system thickness $d$ nor the gravity number affect the long-time scaling.

To identify the acting coarsening modes we illustrate the flow pattern by calculating differences between the interfaces $h\left(x, y, t_{1}\right)$ and $h\left(x, y, t_{2}\right)$ at different times $t_{1}$ and $t_{2}$, respectively, shown for the evolution of drops in Fig.14 (corresponding to Fig.10). One can identify two different coarsening mechanisms being dominant at different times within a single long-time evolution. As a result of the short-time evolution many small drops exist. Neighboring drops attract each other strong enough to move the entire (small) drops and finally combine to one large drop sitting at an intermediate position. This translational coarsening mode is illustrated in Fig.14(a) where its signature in the difference plot is that all drops have white (mass gain) and black (mass loss) parts of their edges.

For larger times a transition from the dominant translational mode to a dominance of the volume transfer mode takes places. Now the mean distance of the drops is too large to get the (large) drops moving. Only material is transported between the sitting drops resulting in a slow disappearance of smaller drops and the growth of the larger drops. This mass transfer mode is illustrated in Fig.14(b) where its signature in the difference plot is that there exist drops that have completely white or completely black edges. 
Finally, we want to show that our descriptive explanation for the viscous timescales (and therefore for the interface velocity directions) given above in Section with the fully nonlinear evolution. We integrated Eq. (11) numerically in two dimensions for a RT unstable system with $H_{1}=H_{2}=0$ (integration was stopped before film rupture occurred). The $x$-components of the velocities $u_{1}(x, z, t)$ and $u_{2}(x, z, t)$ can be calculated from Eq. ([6). They are plotted in Fig.15](right plots) for the position $x=20$. The left panels of Fig.15] display the interfaces and the contour lines of the streamfunction $\varphi\left(u=-\partial_{z} \varphi\right.$, solid lines $\varphi>0$, dashed lines $\varphi<0$ ). For $\mu<\mu_{c}$ (Fig.15(a)) the interface velocity is negative, i.e. fluid moves away from the deformation minimum and therefore the lower layer is the driving layer (solid arrows in Fig.15(a)). For $\mu>\mu_{c}$ (Fig.15(b)) the interface velocity is positive. Hence fluid moves to the deformation minimum and the upper layer is the driving layer (see also solid arrows in Fig.[5(b)).

\section{Thermocapillary effects}

In this section we include thermocapillary effects (using Eq. (24)). Thermocapillarity can act both stabilizing and destabilizing as seen from linear analysis. To avoid an amplification due to gravity we use parameters from the second column in Tab. Therefore, $\rho<1$ and gravity stabilizes the flat film. Eq. (39b) provides then the critical system thickness $d_{c} \approx 3.34$. An unstable initial flat film is obtained for $d>d_{c}\left(d<d_{c}\right)$ by heating from below (above). In the following, we use $d=2$ and $d=4$ to illustrate the destabilization by different directions of heating.

For $d=2$, the critical Marangoni number is $M_{c} \approx-5$ and the system becomes unstable for $M<M_{c}$. Fig. 16 shows a numerical run for $M=-10$. Small initial disturbances evolve smoothly into drops which coarse in the long-time evolution $\left(t=3 \times 10^{5}\right)$ corresponding to 
the prediction of a Maxwell construction (Maxwell point $h_{M} \approx 1.34$ ). Fig.17 (solid line) shows the mean wave number $\langle k\rangle(t)$ and the energy versus time. The energy is again a monotonously decreasing function in time. The mean wave number $\langle k\rangle(t)$ has again two local extrema. The minimum reached at $t_{\text {min }} \approx 1800$ corresponds to the fastest linear wave number. The long-time coarsening sets in after the local maximum at $t_{\max } \approx 4000$ and the scaling coefficient defined in Eq. (43) is determined to be $\beta \approx 0.16$.

For $d=4$ the critical Marangoni number is $M_{c} \approx 37.84$ and we use $M=70$ for the numerical run displayed in Fig.18. Starting from small perturbations one hole evolves rapidly at $t \approx 700$. Subsequently, more and more holes arise $(t=1100)$. For $t>1100$ coarsening sets in and in the long-time limit a single hole remains $\left(t=2 \times 10^{5}\right)$. This corresponds to the prediction of the Maxwell construction (Maxwell point $h_{M} \approx 0.51$ ). The mean wave number $\langle k\rangle$ in Fig.17 (dashed line) shows again a minimum corresponding to the fastest linear wave number and a very pronounced maximum indicating the rapid evolution of one hole between the two extrema. Since the averaging in Eq. (42) gives approximately also the root of the mean curvature the abrupt rise of $\langle k\rangle$ is obvious even for the evolution of a single hole. The long-time scaling is with $\beta \approx 0.27$ remarkably faster than for $d=2$. Note, that we found numerically that the scaling exponent for $d=4$ does nearly not depend on the Marangoni number.

The differences in the short-time evolution for $d=2$ and $d=4$ (rapid evolution of one hole for $d=4$ versus smooth evolution of many drops for $d=2$ ) can be understood in terms of the effective mobilities $Q_{1}^{\prime}(h)=G(1-\rho) Q_{1}(h)$ and $Q_{2}^{\prime}(h)=M Q_{2}(h)$. shown in Fig.[19] For $d=4, Q_{2}^{\prime}$ crosses zero close to $h=1$ (thick lines, $h_{c} \approx 1.2$ from Eq. (14)) and $Q_{1}^{\prime}$ increases for increasing $h$. Therefore, the interface evolution is slowed down for $h>1$ (and finally stopped for $h>h_{c}$ ) and accelerated for $h<1$. This results in a rapid hole evolution. For 
$d=2$, both mobilities show an approximate symmetry around $h=1$ (thin lines). Hence, no interface thickness is suppressed allowing for a smooth evolution of drops.

\section{Application of an electric field}

Finally, we illustrate the time evolution caused by a vertically applied electrical field. We use the parameters from the second column in Tab.\for an isothermal $(M=0)$ system with $G=0$. Fig. 20] shows snapshots of the long-time evolution for an applied voltage $U=30$. Initially small disturbances of the interface evolve smoothly to drops and the long-time coarsening sets in at $t \approx 10000$. The dependence of the mean wave number on time shown as dotted line in Fig.[17 shows a minimum at $t_{\min } \approx 1500$ and a maximum at $t_{\max } \approx 4700$. Again, the minimum coincidences to the wave number of the fastest linear mode $k_{\max } \approx 0.18$.

The derived long-time scaling exponent $\beta \approx 0.04$ is small compared to the exponents measured above for the Rayleigh-Taylor and thermocapillary instabilities. In absolute values we find only a small change from $\langle k\rangle=0.18$ at $t=10^{4}$ to $\langle k\rangle=0.16$ at $t=10^{5}$. We conclude, that one can consider the length scale of the pattern in the long-time evolution as frozen to the value of the wavelength of the fastest linear mode $\left(2 \pi / k_{\max }\right)$ at least up to $t=10^{5}$.

\section{CONCLUSION}

Using long-wave approximation we have derived a single evolution equation for the interface profile of a two-layer system bounded by rigid plates. This equation is written in a general form to facilitate the inclusion of arbitrary body forces and normal or tangential forces at the interface. In the analysis of the model presented here, we have focused on the influences 
of gravity, thermocapillarity and electrostatic fields.

We have shown that the mobility of the normal-stress and the body force terms $Q_{1}(h)$ is always positive as in the one-layer case. However, it tends to zero not only for $h \rightarrow 0$ as for the one-layer system but also for $h \rightarrow d$. The latter has no counterpart in one-layer systems where the mobility increases monotonously with the film thickness $\stackrel{2}{*}$. The second qualitative difference is the sign change of the mobility for the shear-stress term $Q_{2}(h)$. Both mobilities can affect strongly the linear and nonlinear evolution. For instance, the direction of heating needed for destabilization is determined by the zero crossing of the mobility $Q_{2}(h)$. Furthermore, the shapes, zeros and extrema of the mobilities allow at least a qualitative prediction of the dynamics of the system without any numerical investigation. Moreover, in contrast to weakly nonlinear theories ${ }^{24}$ we are able to check these descriptive criterions integrating the fully nonlinear equation numerically. A non-smooth rapid hole (drop) evolution in-between the short-time and long-time regime can already be estimated from the trend of the mobilities.

Remarkably, although in the heated case the system is dissipating energy through convection within the drops (or around the holes) even when the final stable state is reached, the use of long-wave approximation allows for a variational formulation using an energy or Lyapunov functional for the film thickness profile. The film thickness evolution equation takes then the form of the simplest possible equation for the dynamics of a conserved order parameter field ${ }^{36.37 .38}$. A prominent representative of this class of systems is the Cahn-Hilliard equation describing the evolution of a concentration field for a binary mixture ${ }^{39}$. In contrast to the one-layer case $\frac{11.37}{}$, here the energy itself depends on material parameters that characterize the dynamics of the system, namely the ratio of viscosities. We used the energy functional to predict the expected long-time behavior, namely the evolution of holes, drops or maze 
structures. It also allows for the study of metastable states. The predictions have been confirmed by fully nonlinear numerical integrations of the evolution equation.

Using a linear stability analysis we have discussed the conditions for a gravitational or Rayleigh-Taylor instability, thermocapillary destabilization and stabilization, and an electrohydrodynamic instability for dielectric liquids under the influence of an electrical field. We have shown that thermocapillarity may act stabilizing as well as destabilizing depending on material parameters. The behavior becomes intuitively clear because when treating both layers in the same way no direction of heating should be preferred. This implies that depending on material parameters both ways to destabilize the system - heating from above and heating from below - have to be possible.

We have given special emphasis to the study of the possibility to stabilize a RayleighTaylor unstable two-layer system by heating from below. This seemingly counter-intuitive behavior first discussed in Ref. ${ }^{24}$ is a typical property of multi-layer systems and is directly correlated with the change of sign of the mobility $Q_{2}(h)$. However, we have shown that the stabilized Rayleigh-Taylor system is metastable. This explains a problem encountered in the experiments of BURGESS ET AL. 21. Although they could stabilize a Rayleigh-Taylor unstable 'oil on air' system by heating from below this was only possible in ten percent of the experimental runs. This is due to the fact that the preparation of the initial flat film involved large amplitude disturbances. Because of the metastability of the system this results in the destabilization of ninety percent of the runs because the disturbance is larger than the critical one. The transition from the two- to the three-dimensional equation has shown that a weak mean flow arises. We neglect this additional flow in the main part of the present work due to its very weak influence on the presented results. However, the mean flow has been discussed in detail in the appendix. 
We have implemented numerical schemes for two- and three-dimensional versions of the fully nonlinear equation and have given an overview showing different possible long-time evolutions consisting of coarsening hole, drop or maze patterns. Furthermore, we have analysed the scaling behavior by calculating the time dependence of the mean wave number of the patterns and extraction of the tendency of scaling exponents.

Isothermal two-layer systems, i.e. taking into account gravitation and disjoining pressures only, show the same long-time scaling for different system thicknesses $d$. However, incorporating thermocapillarity the system thickness $d$ affects the long-time scaling essentially. This scaling behavior is in contrast to the one-layer case which was found to be determined by one scaling exponent $(\beta \approx 0.21)^{10}$. The one-layer coefficient lies within the two-layer range $0.16 \leq \beta \leq 0.27$ found here. Moreover, an isothermal $(M=0)$ and agravic $(G=0)$ electrohydrodynamically unstable system reveals a very small scaling coefficient $(\beta \approx 0.04)$. To our knowledge for this class of evolution equations such a slow long-time scaling is found for the first time.

Finally, we have shown that tangential interface forces (thermocapillary forces) allow for rapid hole (or drop) formation after the short-time evolution. Again, this mechanism can be understood in terms of the change of sign of the tangential mobility $Q_{2}(h)$.

In the present work, disjoining pressures were solely used to inhibit rupture of the layers to allow for the study of the long-time behavior. This corresponds to the assumption that liquid 1 and 2 wet the lower and upper plate, respectively. However, our present theory is not apt to describe situations where both layers are ultrathin (less than $100 \mathrm{~nm}$ ), a situation gaining more and more importance for research communities and industrial applications. Then disjoining pressures dominate and the used terms are not exact enough because part of the forces between the lower (upper) fluid and the upper (lower) substrate are neglected. 
Furthermore, the used Van der Waals interaction should be supplemented by additional short-range interactions $\frac{13}{}$. A further analysis of disjoining pressures in two-layer systems seems promising and will be published elsewhere.

We emphasize our results for the action of a vertical electric field since recent experiments focus on such systems, as for example, done by LIN ET AL. using two polymeric liquids ${ }^{30.31}$. They monitored the time evolution up to the impingement of the lower polymer layer on the upper electrode and showed a series of snapshots of the evolving morphology (Fig. 4 of Ref. $\underline{30}$ ). Interestingly, they found a nearly constant length scale of the evolving columnar structures from the early stage on, corresponding to the fastest linear mode. This corresponds to the results of our linear and nonlinear analysis of this case that we performed for a comparable ratio of permittivities. Furthermore, a visible concordance of both timeseries exists. The small scaling exponent $\beta \approx 0.04$ found here can be regarded as a structure length frozen to the fastest linear wavelength. This indicates, that our model gives reasonable results even for macromolecular liquids.

Finally, we stress the advantages for physics as well as industrial applications of bounded two-layer systems. From a physical point of view our single interface equation captures both the long-wave evolution and the interface interactions of two fluids. Therefore it allows for detailed investigations how the fluid properties of both the upper and the lower fluid layer determine the stability, metastability and short-time as well as long-time evolution. Furthermore ultrathin films play already a major role to create desired structures or stable flat interfaces. Usually one-layer equations are used for that kind of industrial applications. However, controlled boundary conditions, well defined bulk properties and consequently well defined interface actions enhance the accuracy of experiments as well as the examination of theory and experiment. 


\section{APPENDIX: THREE-DIMENSIONAL EVOLUTION EQUATION}

The generalization of the derivation of the film thickness equation to three dimensions is straightforward up to Eq. (8) for which the three-dimensional (already integrated) version reads

$$
\nabla \cdot\left[A(h)\left(\nabla \tilde{P}_{1}-F_{1}(h) \nabla(\mathcal{N}+\Phi)-F_{2}(h) \overrightarrow{\mathcal{T}}\right)\right]=0
$$

where $\nabla=\left(\partial_{x}, \partial_{y}\right)$ and

$$
A(h)=-\frac{D}{12 \mu((\mu-1) h+d)}
$$

with $D$ from Eq. (10c).

Eq. (A.1) cannot be solved analytically for the pressure $\tilde{P}_{1}$. However, to fulfill Eq. (A.1) its argument must be of the form

$$
\frac{1}{A(h)} \operatorname{rot}\left(f \vec{e}_{z}\right)=\nabla \tilde{P}_{1}-F_{1}(h) \nabla(\mathcal{N}+\Phi)-F_{2}(h) \overrightarrow{\mathcal{T}}
$$

with $f$ as an arbitrary scalar function, $\operatorname{rot}\left(f \vec{e}_{z}\right)=\left(\partial_{y} f,-\partial_{x} f, 0\right)$ and $F_{1}(h)$ from Eq. (10a). Note that this function $f$ is already present without symmetry breaking effects (e.g. inclined or rotating systems). Substitution of the gradient pressure from Eq. (A.3) in the three dimensional correspondent to Eq.(17) provides the evolution equation for $h$

$$
\partial_{t} h=\nabla \cdot\left[Q_{1}(h) \nabla(\mathcal{N}+\Phi)+Q_{2}(h) \overrightarrow{\mathcal{T}}\right]+\nabla \cdot\left[Q_{3}(h) \operatorname{rot}\left(f \vec{e}_{z}\right)\right]
$$

where $f$ as a function of $h$ and its spatial derivatives is given implicitly by

$$
\begin{aligned}
-\frac{1}{A(h)} \triangle f= & \partial_{h}\left(\frac{1}{A(h)}\right) \operatorname{rot}\left(h \vec{e}_{z}\right) \cdot \operatorname{rot}\left(f \vec{e}_{z}\right)+\partial_{h} F_{1}(h) \operatorname{rot}\left(h \vec{e}_{z}\right) \cdot \nabla(\mathcal{N}+\Phi) \\
& +\partial_{h} F_{2}(h) \operatorname{rot}\left(h \vec{e}_{z}\right) \cdot \overrightarrow{\mathcal{T}}+F_{2}(h) \operatorname{rot}(\overrightarrow{\mathcal{T}}) \cdot \vec{e}_{z}
\end{aligned}
$$

resulting from applying the curl to Eq. (A.3). 
The third mobility

$$
Q_{3}(h)=F_{1}(h)-1
$$

is a monotonically decreasing function in $h\left(Q_{3}(0)=0, Q_{3}(d)=-1\right)$. Note that $\partial_{h} Q_{3}(h)=$ $\partial_{h} F_{1}(h)$ is zero for $h=0$ and $h=d$ and negative for $0<h<d$. The additional function $f$ reflects the possible mean flow of the system induced by a vertical vorticity contribution. We mention that in the one-layer limit $(\mu \rightarrow 0$ or $d \rightarrow \infty)$ this vorticity contribution is zero $(1 / A(h) \rightarrow 0)$ and the usual normal condition for the pressure is recovered.

Substituting $\mathcal{N}, \Phi$ and $\mathcal{T}$ (from subsection $\llbracket$ B ) in the rhs of Eq. (A.4b) provides

$$
\frac{1}{A(h)} \triangle f+\partial_{h}\left(\frac{1}{A(h)}\right) \operatorname{rot}\left(h \vec{e}_{z}\right) \cdot \operatorname{rot}\left(f \vec{e}_{z}\right)=C^{-1} \partial_{h} F_{1}(h) \operatorname{rot}\left(h \vec{e}_{z}\right) \cdot \nabla(\triangle h)
$$

showing that solely the surface tension contributes to $f$.

Due to the properties of $\partial_{h} F_{1}(h)$ the vorticity $f$ is basically determined by the geometrical shape of the interface. It can be shown with Eq. (A.6) that rotationally symmetric structures are not affected in the strongly nonlinear regime by this additional vorticity contribution. Furthermore Eq. (A.6) reveals that for small deviations from symmetric states the vorticity $f$ acts in the same direction as surface tension itself in Eq. A.4a .

We have checked and confirmed all numerical runs of this paper taking into account Eq. (A.6). In general the contribution of $f$ is small. Therefore we neglect it and consider the evolution equation (12) only. However, we note that the effects of Eq. (A.6) on the evolution equation are an attractive and important subject for future investigations. 
1 O. Reynolds, On the theory of lubrication and its application to Mr.Beauchamp Tower's experiments, Philos. Trans. R. Soc. London 177, 157 (1886)

2 A. Oron, S.H. Davis and S.G. Bankoff, Long-scale evolution of thin liquid films, Rev. Mod. Phys. 69, $931(1997)$

3 L.E. Scriven and C.V. Sternling, On cellular convection driven by surface tension gradients, J. Fluid Mech. 19, 321 (1964)

4 D.A. Goussis and R.E. Kelly, Surface wave and thermocapillary instabilities in a liquid film flow, J. Fluid Mech. 223, 25 (1991)

5 A.A. Golovin, A.A. Nepomnyashchy and L.M. Pismen, Interaction between short-scale Marangoni convection and long-scale deformational instability, Phys. Fluids 6, 34 (1994)

6 J.P. Burelbach, S.G. Bankoff and S.H. Davis, Nonlinear stability of evaporating/condensing liquid films, J. Fluid Mech. 195, 463 (1988)

7 R.J. Deissler and A. Oron, Stable localized patterns in thin liquid films, Phys. Rev. Lett. 68, $2948(1992)$

8 A. Oron and P. Rosenau, On a nonlinear thermocapillary effect in thin liquid layers, J. Fluid Mech. 273, 361 (1994)

9 A. Oron, Nonlinear dynamics of three-dimensional long-wave Marangoni instability in thin liquid films, Phys. Fluids 12, 1633 (2000)

10 M. Bestehorn, A. Pototsky and U. Thiele, 3D Large scale Marangoni convection in liquid films, Eur. Phys. J. B 33, 457 (2003)

11 U. Thiele and E. Knobloch, Thin liquid films on a slightly inclined heated plate, Physica D 190, 
$213(2004)$

12 E. Ruckenstein and R. Jain, Spontaneous rupture of thin liquid films, J. Chem. Soc. Faraday Trans. II 70, $132(1974)$

13 J.N. Israelachvili, Intermolecular and surface forces, Academic Press, London (1992)

14 U. Thiele, Open questions and promising new fields in dewetting, Eur. Phys. J. E 12, 409 (2003)

15 K.D. Danov, V.N. Paunov, N. Alleborn, H. Raszillier and F. Durst, Stability of evaporating twolayered liquid film in the presence of surfactant-I. The equations of lubrication approximation, Chem. Eng. Sci. 53, 2809, (1998)

16 K.D. Danov, V.N. Paunov, S.D. Stoyanov, N. Alleborn, H. Raszillier and F. Durst, Stability of evaporating two-layered liquid film in the presence of surfactant-II. Linear analysis, Chem. Eng.

Sci. 53, 2823, (1998)

17 V.N. Paunov, K.D. Danov, N. Alleborn, H. Raszillier and F. Durst, Stability of evaporating two-layered liquid film in the presence of surfactant-III. Non-linear stability analysis, Chem. Eng. Sci. 53, 2839, (1998)

18 A. Pototsky, M. Bestehorn, D. Merkt and U. Thiele, Alternative pathways of dewetting for a thin liquid two-layer film, Phys. Rev. E 70, 025201, (2004)

19 S.G. Yiantsios and B.G. Higgins, Rayleigh-Taylor instability in thin viscous films, Phys. Fluids A 1, $1484(1989)$

20 S.J. VanHook, M.F. Schatz, J.B. Swift, W.D. McCormick and H.L. Swinney, Long-wavelength surface-tension-driven Bénard convection: experiment and theory, J. Fluid Mech. 345, 45 (1997)

21 J.M. Burgess, A. Juel, W.D. McCormick, J.B. Swift and H.L. Swinney, Suppression of Dripping from a Ceiling, Phys. Rev. Lett. 86, 1203 (2001)

22 K.A. Smith, On convective instability induced by surface-tension gradients, J. Fluid Mech. 24, 
$401(1966)$

I.B. Simanovskii and A.A. Nepomnyashchy, Convective instabilities in systems with interface, Gordon and Breach, Amsterdam (1993)

24 A.A. Nepomnyashchy and I.B. Simanovskii, Long-wave thermocapillary convection in layers with deformable interfaces, Appl. Math. Mech. 54, 490 (1990)

25 B.S. Tilley, S.H. Davis and S.G. Bankoff, Nonlinear long-wave stability of superposed fluids in an inclined channel, J. Fluid. Mech. 277, 55 (1994)

26 S.R. Majumdar and M.E. O'Neill, Vertical Boundary Effects on the Electrohydrostatic Instability of a Fluid Interface, J. Inst. Maths Applics 17, 343 (1976)

27 A.A. Mohamed, E.F. Elshehawey and M.F. El-Sayed, Electrohydrodynamic Stability of Two Superposed Viscous Fluids, J. Colloid Interface Sci. 169, 65 (1995)

28 J.R. Melcher and C.V. Smith, Electrohydrodynamic Charge Relaxation and Interfacial Perpendicular-Field Instability, Phys. Fluids 12, 778 (1969)

${ }^{29}$ K. Savettaseranee, D.T. Papageorgiou, P.G. Petropoulos and B.S. Tilley, The effect of electric fields on the rupture of thin viscous films by van der Waals forces, Phys. Fluids 15, 641 (2003)

30 Z. Lin, T. Kerle, T. P. Russell, E. Schäffer and U. Steiner, Structure Formation at the Interface of Liquid/Liquid Bilayer in Electric Field, Macromolecules 35, 3971 (2002)

31 Z. Lin, T. Kerle, S.M. Baker, D.A. Hoagland, E. Schäffer, U. Steiner and T.P. Russell, Electric field induced instabilities at liquid/liquid interfaces, J. Chem. Phys. 114, 2377 (2001)

32 M.B. Williams and S.H. Davis, Nonlinear theory of film rupture, J. Colloid Interface Sci. 90, $220(1982)$

33 L.D. Landau and E.M. Lifschitz, Electrodynamics of continuous media, Akademie Verlag Berlin (1990) 
34 O. Penrose and P.C. Fife, Thermodynamically consistent models for phase-field type for the kinetics of phase transitions, Physica D 43, 44 (1990)

35 A. Engel and J.B. Swift, Planform selection in two-layer Bénard-Marangoni convection, Phys. Rev. E 62, 6540 (2000)

36 V.S. Mitlin, Dewetting of solid surface: analogy with spinodal decomposition, J. Colloid Interface Sci. 156, $491(1993)$

37 A. Oron and P. Rosenau, Formation of patterns induced by thermocapillarity and gravity, J. Physique II France 2, 131 (1992)

38 J.S. Langer, p. 297 in Solids far from Equilibrium, Ed. C. Godreche, Cambridge University Press, (1992)

39 J.W. Cahn, Phase Separation by Spinodal Decomposition in Isotropic Systems, J. Chem. Phys. 42, $93(1965)$ 


\begin{tabular}{|l|r|r|}
\hline Fluid 2 - Fluid 1 & HT70 - silicon oil 5cS & silicon oil 5cS - HT70 \\
\hline density $\rho=\rho_{2} / \rho_{1}$ & 1.826 & 0.548 \\
\hline viscosity $\mu=\mu_{2} / \mu_{1}$ & 0.1826 & 5.48 \\
\hline thermal conductivity $\lambda=\lambda_{2} / \lambda_{1}$ & 0.598 & 1.67 \\
\hline permittivity $\varepsilon=\varepsilon_{2} / \varepsilon_{1}$ & 0.77 & 1.3 \\
\hline
\end{tabular}

TABLE I: Material parameters for a silicon oil 5cS - HT70 system taken from Ref. $\frac{35}{3}$. The values in the first column are for HT70 on silicon oil 5cS, whereas for the second column the liquids are interchanged, i.e. silicon oil 5cS on HT70. 
Figure captions:

Fig.1. Sketch of the system. The two layered immiscible liquids are bounded by two rigid smooth plates. The flat interface is situated at the mean height $h_{0}$. The position $h(x, y, t)$ of an evolving interface profile is a function of $x, y$ and $t$ only.

Fig.2. Shown are the mobilities $Q_{1}(h)$ (normal-stress and body force terms) and $Q_{2}(h)$ (tangential-stress term) for $d=1.3$ and $\mu=0.1826$. The mobility $Q_{1}(h)$ is always positive and vanishes for $h \rightarrow 0$ and $h \rightarrow d$. The mobility $Q_{2}(h)$ changes sign at $h_{c} \approx 0.91$

Fig. 3. The dependence of the linear growth rate $\chi$ on wavenumber $k$ for $d=1.3, \rho=1.826$, $\mu=0.1826$ and $\lambda=0.598$ (parameters from the first column in Tab.【一) at $G=1$ and $C^{-1}=20\left(H_{1}=H_{2}=0\right)$. In the non-isothermal case $M= \pm 1$.

Fig.4. The stability diagrams show the critical Marangoni number $M_{c}$ in dependence of the ratio of viscosities $\mu$ for $d=1.3, \lambda=0.598$ and $G=5\left(H_{1}=H_{2}=0\right) . \quad M_{c}>0$ $\left(M_{c}<0\right)$ corresponds to heating from below (above). The direction of heating leading to destabilization changes at the critical viscosity $\mu_{c}=0.09$. The left (right) panel corresponds for $M=0$ to a Rayleigh-Taylor unstable (stable) system with $\rho=1.826>$ $1(\rho=0.548<1)$.

Fig.5. Sketch illustrating the mechanism of the (a) destabilizing and (b) stabilizing thermocapillary action for a Rayleigh-Taylor (RT) unstable system heated from below. Solid arrows indicate the liquid velocity close to the interface in the respective driving layer for pure RT, dashed arrows signal the effect of thermocapillarity. (a) Due to thermocapillarity the total flow in the driving layer is directed away from the deformation as 
known from one-layer systems. This causes an amplification of the disturbance. (b) The viscous timescale $\tau_{\mu_{2}} \propto\left(d-h_{0}\right)^{2} / \mu_{2}$ of the upper layer is faster than the one of the lower layer. The influence of the upper driving layer dominates leading to flow to the deformation minimum. Thermocapillarity causes flow in the opposite direction, thereby weakening or completely damping the disturbance.

Fig.6] Shown is the critical Marangoni number $M_{c}$ versus the system thickness $d$ for $\rho=0.548$, $\mu=5.48, \lambda=1.67$ and $G=5$. The critical system thickness is $d_{c} \approx 3.34$. For $d<d_{c}$ heating from above acts destabilizing and $M_{c}$ is a monotonic function of $d$. For $d>d_{c}$ heating from below destabilizes and a minimum results from competing mechanisms (see main text). Inclusion of stabilizing disjoining pressures cause a maximum of $M_{c}$ for $d<d_{c}$ but have nearly no influence for $d>d_{c}$ (dashed lines).

Fig.7] Maxwell constructions (horizontal lines) based on the local energy are given for (a) three different system thicknesses $d$ (see legend) of a Rayleigh-Taylor unstable system at $G=5, C^{-1}=20, H_{1}=H_{2}=0.01, M=0, \rho=1.826$ and $\mu=0.1826$. For $d=2$, the Maxwell point is 1 (vertical dotted line). $d>2$ leads to drop solutions (dashed line). Hole solutions are expected for $d<2$ (solid line). (b) Illustrates the occurrence of metastable states using $G=10, H_{1}=0.1, H_{2}=0.01, \rho=1.826$, and $\mu=0.1826$, i.e. an Rayleigh-Taylor unstable system. The isothermal case (dashed line) is linearly unstable, whereas heating from below $(M=10$, solid line) stabilizes the system linearly. However, the local maximum still exists at $h<1$ indicating a metastable flat film.

Fig.8. Shown is the critical Marangoni number $M_{c}$ versus system thickness $d$ for $G=10$, $C^{-1}=20, \rho=1.826, \mu=0.1826, \lambda=0.598, H_{1}=0.1$ and $H_{2}=0.01$. The isothermal 
system is Rayleigh-Taylor unstable $(M=0)$. For $d>d_{c}\left(d<d_{c}\right)$ heating from above (below) stabilizes the system. However, for the shown range of $M$ the system remains metastable, i.e. finite disturbances larger than a critical nucleus will grow.

Fig.9] Snapshots from of the nonlinear evolution of the interface for $d=1.3, G=10$, $C^{-1}=20, \Delta t=0.1, \Delta x=0.5, \rho=1.826, \mu=0.1826, \lambda=0.598, H_{1}=0.1$ and $H_{2}=0.01$. Without heating $(M=0)$ the system is linearly Rayleigh-Taylor unstable implying the growth of infinitely small disturbances (dashed line). For $M>M_{c}=4.62$ the system is metastable. We applied for $M=10>M_{c}$ a strong disturbance of the interface $(h \approx 0.7 \pm 0.1)$ in the vicinity of $x=50$.

Fig.10. Three-dimensional snapshots of the long-time evolution of a Rayleigh-Taylor unstable system at $d=3, G=5, C^{-1}=20, \rho=1.826, \mu=0.1826$ and $H_{1}=H_{2}=0.01$. The system size is $L_{x}=L_{y}=200$ with a resolution $\Delta t=0.1, \Delta x=\Delta y=2$. Initial (small) perturbations of the flat interface lead to drop formation, and subsequent long-time coarsening. Finally, one single drop survives (not shown).

Fig.11] Three-dimensional snapshots of the long-time evolution of a Rayleigh-Taylor unstable system at $d=1.3, G=20, C^{-1}=20, \rho=1.826, \mu=0.1826$ and $H_{1}=H_{2}=0.01$. The system size is $L_{x}=L_{y}=100$ with a resolution $\Delta t=0.1, \Delta x=\Delta y=0.5$. From initially small perturbations holes start to evolve $(t=150)$ smoothly. Subsequently long-time coarsening sets in at $t \approx 1000$ and finally an one-hole solution is reached $\left(t>2 \times 10^{5}\right)$.

Fig.12 Three-dimensional snapshots of the long-time evolution of a maze structure in a Rayleigh-Taylor unstable system at $d=2, G=5, C^{-1}=20, \rho=1.826, \mu=0.1826$ and $H_{1}=H_{2}=0.01$. The system size is $L_{x}=L_{y}=200$ with a resolution $\Delta t=0.1$, 
$\Delta x=\Delta y=2$. It is clearly visible that neither holes nor drops are energetically preferred. In the long-time evolution $(t>1000)$ the usual coarsening takes places.

Fig.13] Shown are (a) the mean wavenumber $\langle k\rangle$ and (b) the energy $E$ in dependence on time for Rayleigh-Taylor unstable systems with $\rho=1.812, \mu=0.1826, H_{1}=H_{2}=0.01$, and different thicknesses $d$ as given in the legend. Horizontal thin lines give the corresponding fastest linear wave numbers.

Fig.14. Grey-level plots of the interface height $h$ at two timesteps and the difference of the two images for the time evolution in Fig.10, In the difference plot dark (light) areas indicate mass loss (gain) of the lower layer (a) During the first stage of the longtime evolution neighboring drops move towards each other to merge indicating the dominance of the translational mode of coarsening. (b) At a later stage, small drops shrink and neighboring large drops grow, indicating the dominance of the mass transfer mode of coarsening.

Fig.15] Given are (left) film thickness profiles $h(x, t)$ and (right) $x$-components of the fluid velocity $u(x=20, z, t)$ for $G=5, C^{-1}=20, \rho=1.826, \mu=0.1826, H_{1}=H_{2}=0$ and a resolution $\Delta t=0.1, \Delta x=0.5$. The left panels also show contour lines of the streamfunction $\varphi\left(u=-\partial_{z} \varphi\right)$. (a) In a system where $\mu<\mu_{c}(d=3)$ at $t=120$ the interface height $h(20, t)<1$ and $\partial_{x} h(20, t)<0$ provides $u<0$ at the interface. The direction of the interface velocity corresponds to the solid arrows in Fig.[5(a), i.e. the lower layer is the driving layer. (b) If $\mu>\mu_{c}(d=1.3)$, at $t=1200$ the interface velocity $u$ at $x=20$ is positive and the upper layer is the driving layer (compare to solid arrows in Fig.罒(b)).

Fig.16. Three-dimensional snapshots of the long-time evolution of a Marangoni instability for 
$M=-10, d=2, G=5, C^{-1}=20, \rho=0.548, \mu=5.48, \lambda=1.671, H_{1}=0.01$ and $H_{2}=0.05$. The system size is $L_{x}=L_{y}=100$ with a resolution $\Delta t=0.1$, $\Delta x=\Delta y=1$. From initially small perturbations the systems evolves smoothly to a single drop solution in the long-time limit $\left(t>10^{6}\right.$, not shown).

Fig.17 Shown are (a) the mean wavenumber $\langle k\rangle$ and (b) the energy $E$ in dependence on time for two thermocapillary and one electrohydrodynamic unstable system (see legend) with $\rho=0.548, \mu=5.48$ and $\lambda=1.671$. Horizontal thin lines give the corresponding fastest linear wave numbers.

Fig.18 Three-dimensional snapshots of the long-time evolution of a Marangoni instability for $M=70, d=4, G=5, C^{-1}=20, \rho=0.548, \mu=5.48, \lambda=1.671, H_{1}=0.05$ and $H_{2}=0.01$. The system size is $L_{x}=L_{y}=200$ with a resolution $\Delta t=0.1$, $\Delta x=\Delta y=1$. We started with initially small perturbations. At $t \approx 700$ one hole starts to evolve rapidly and subsequently more and more holes arise. At $t \approx 1100$ longtime coarsening sets in and continues until a single large hole is reached $\left(t>2 \times 10^{5}\right)$.

Fig.19] Shown are the rescaled mobilities $Q_{1}^{\prime}=G(1-\rho) Q_{1}$ and $Q_{2}^{\prime}=M Q_{2}$ for thermocapillary unstable systems with $d=2, M=70$ (thin lines, numerical run in Fig.16) and $d=$ $4, M=-10$ (thick lines, numerical run in Fig.18). For $d=4$ the zero crossing of $Q_{2}^{\prime}$ is at $h \approx 1.2$. This leads to a suppressed interface evolution for $h>1$ resulting in a rapid hole evolution. For $d=2$ smooth drop evolution results since the regions $h>1$ and $h<1$ are roughly symmetric.

Fig.20] Three-dimensional snapshots of the long-time evolution of an electrohydrodynamic instability for $d=4, C^{-1}=20, U=30, \varepsilon=1.3, \mu=5.48$ and $H_{1}=H_{2}=0.01$. The system size is $L_{x}=L_{y}=300$ with a resolution $\Delta t=0.1, \Delta x=\Delta y=3$. One finds a 
smooth short-time evolution of drops. The long-time coarsening sets in at $t \approx 10000$ and the long-time scaling exponent is very small. 


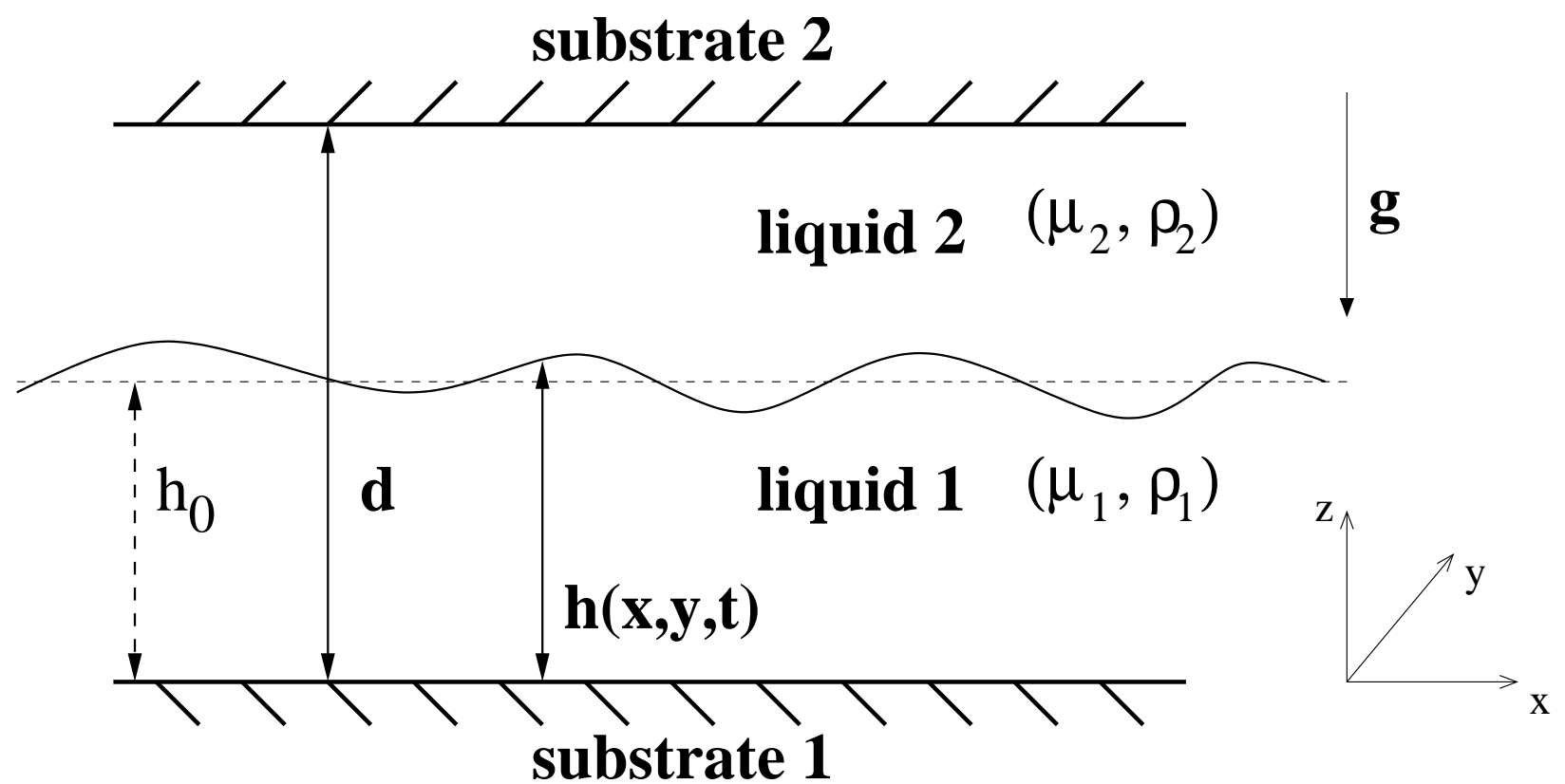

FIG. 1: D.Merkt, Physics of Fluids 


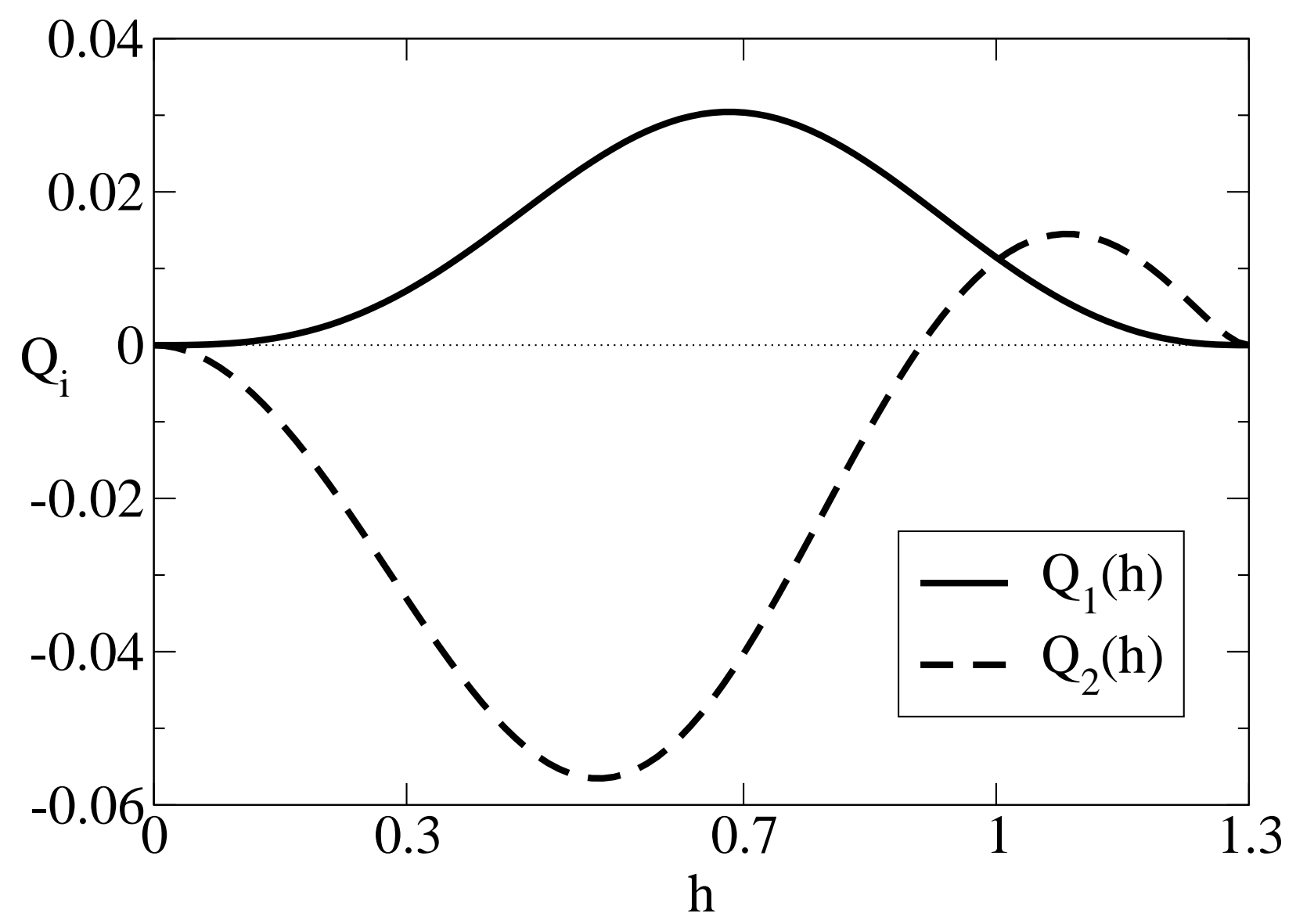

FIG. 2: D.Merkt, Physics of Fluids 


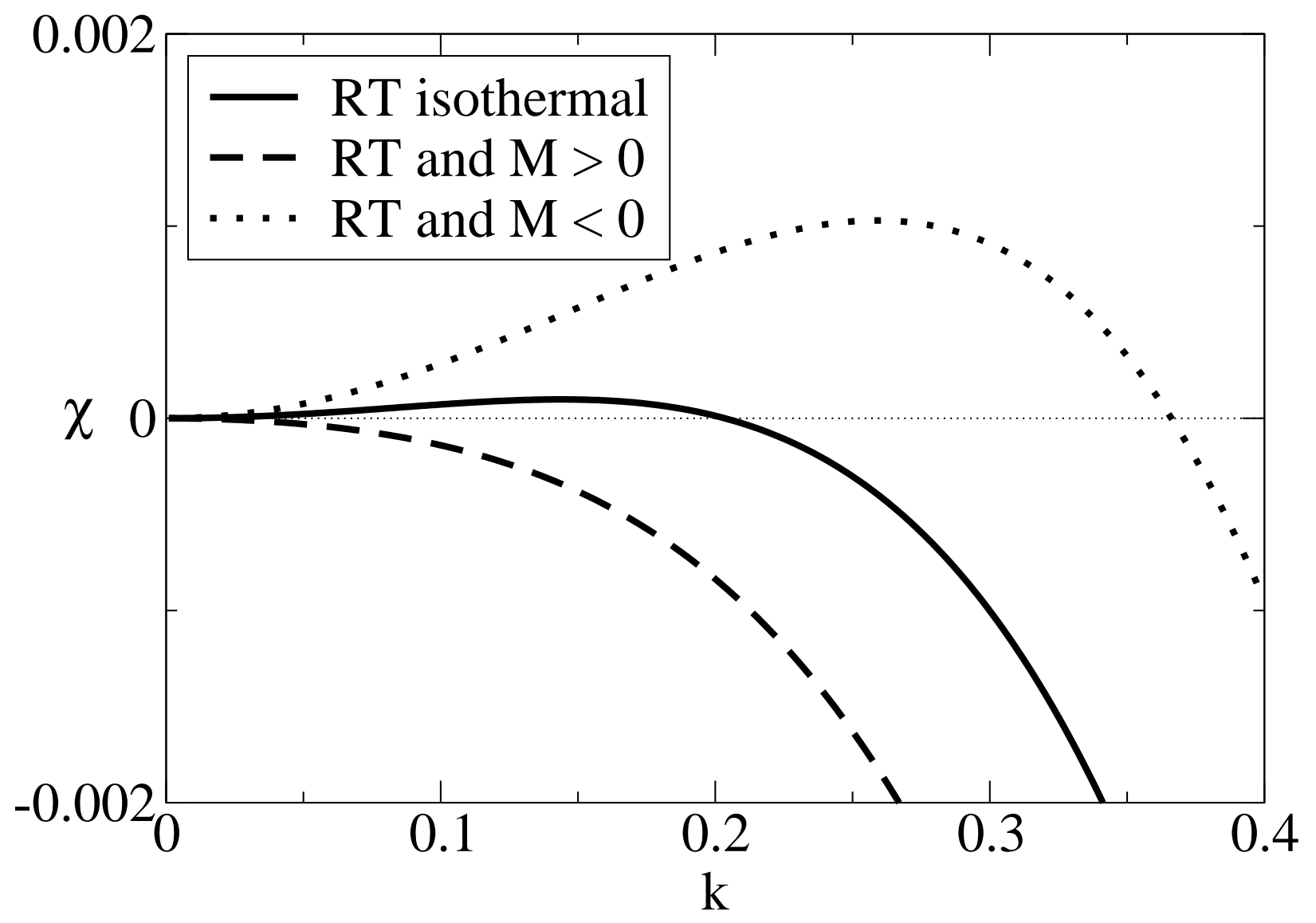

FIG. 3: D.Merkt, Physics of Fluids 

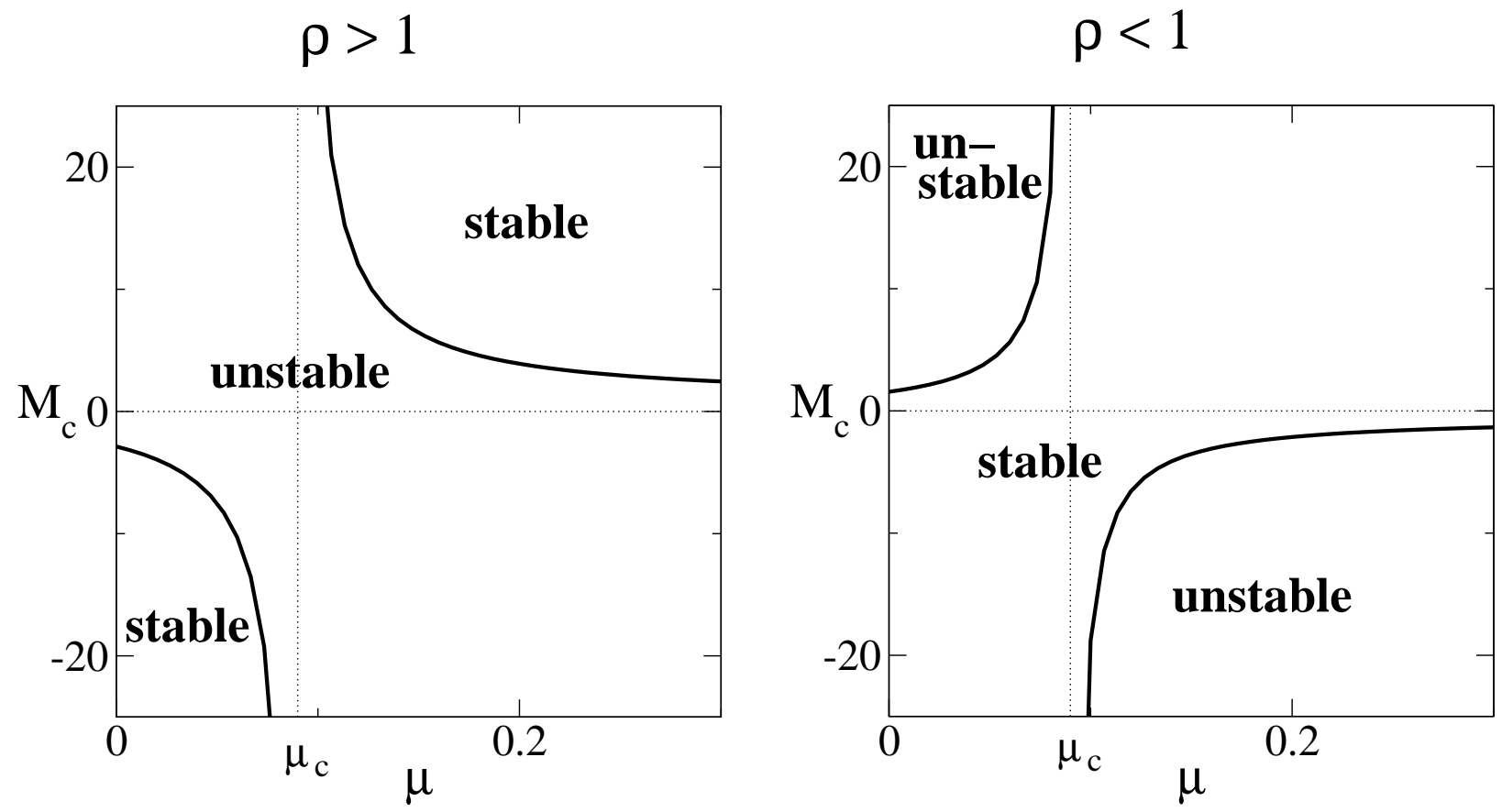

FIG. 4: D.Merkt, Physics of Fluids 
a) $\mu<(d-1)^{2}$

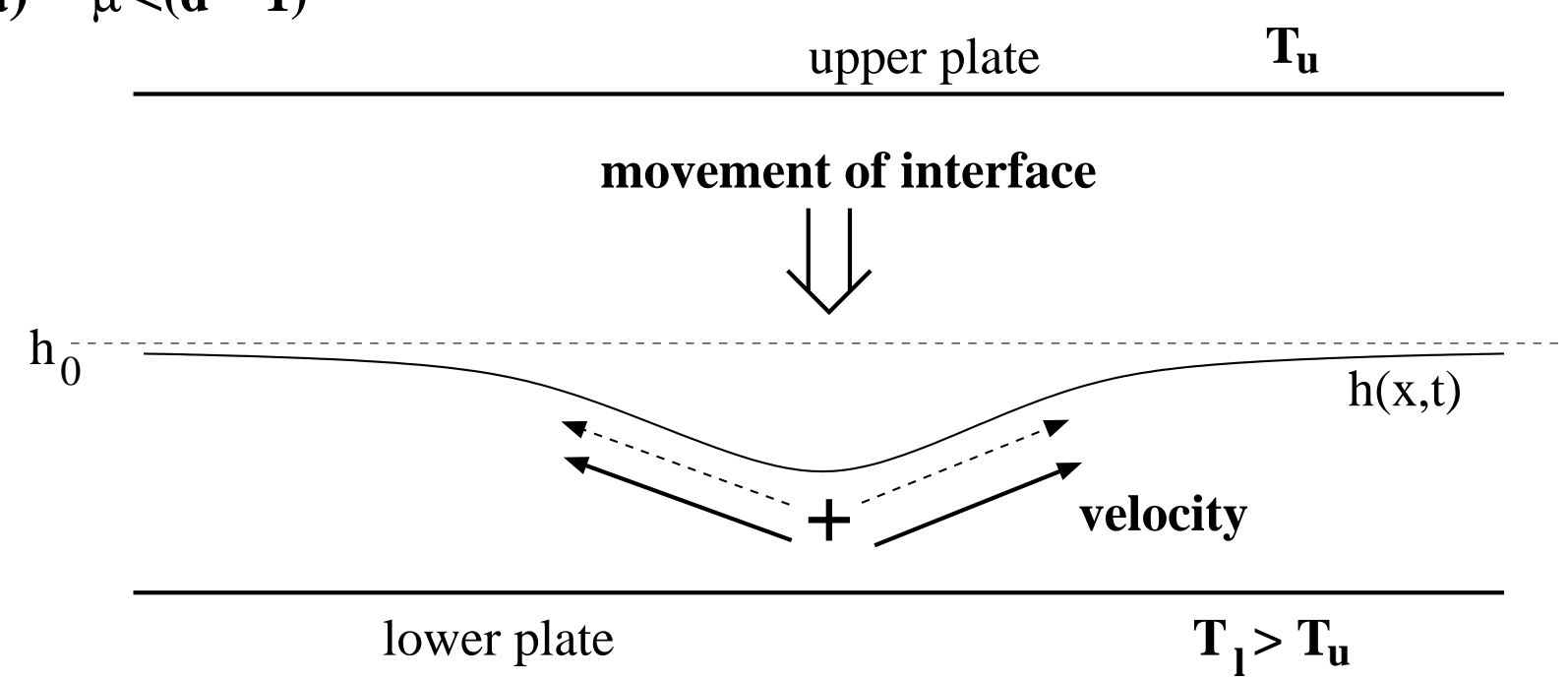

b) $\mu>(d-1)^{2}$

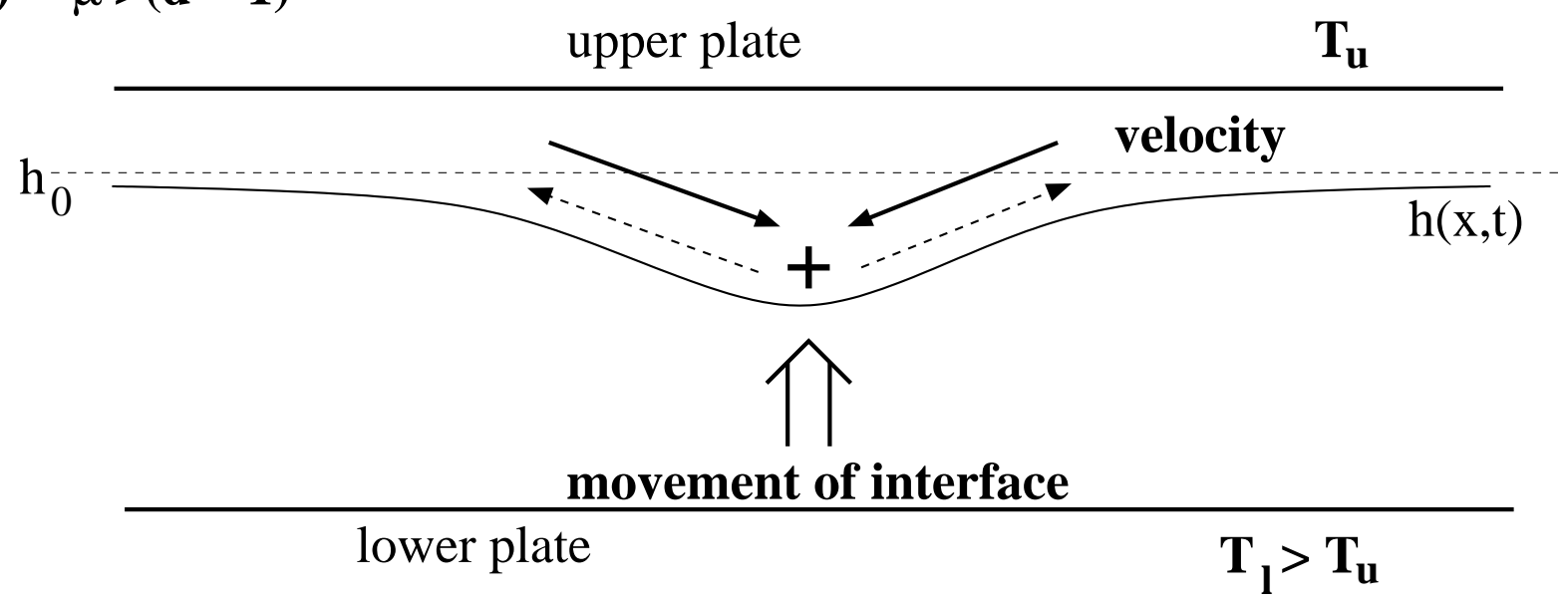

FIG. 5: D.Merkt, Physics of Fluids 


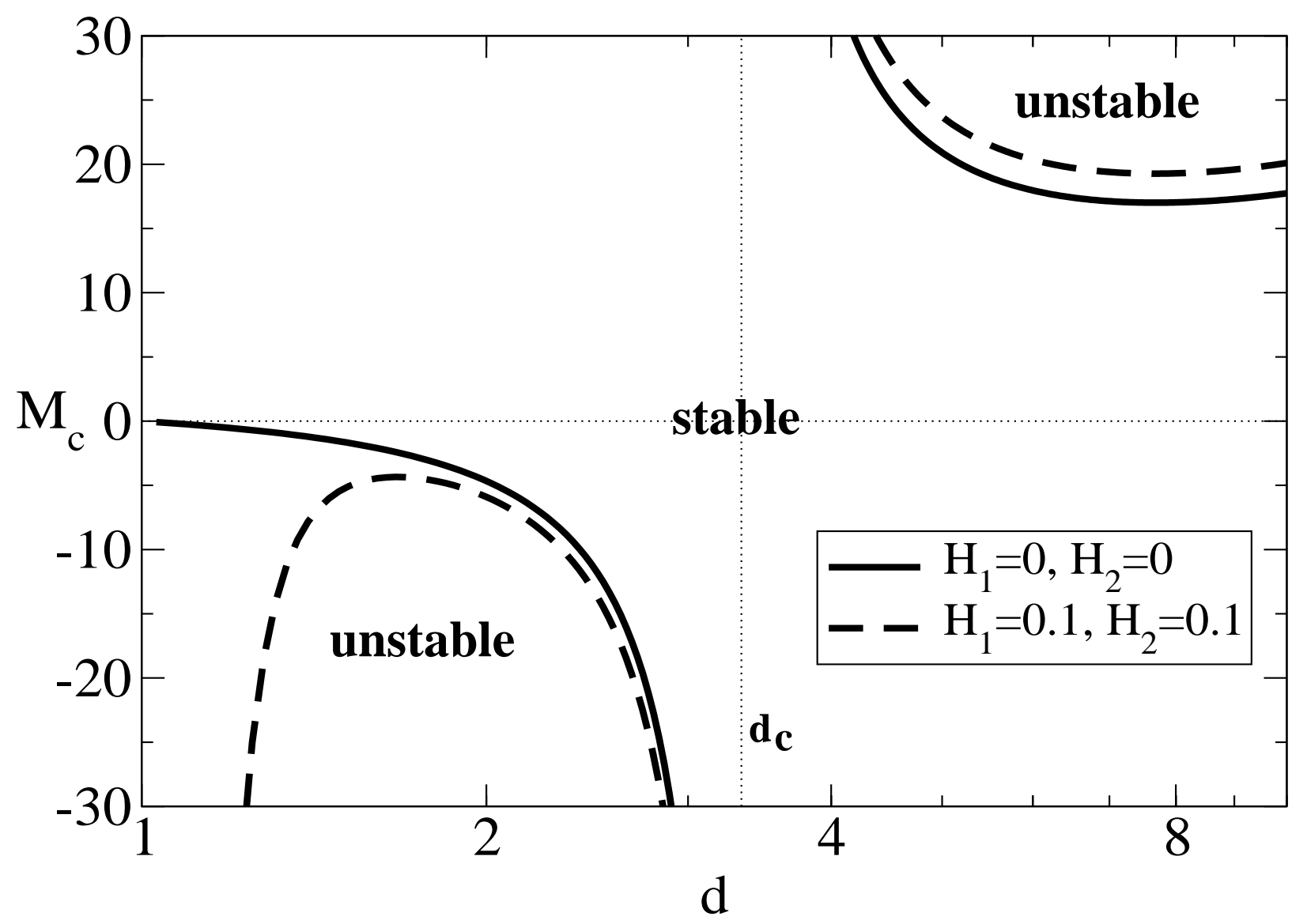

FIG. 6: D.Merkt, Physics of Fluids 

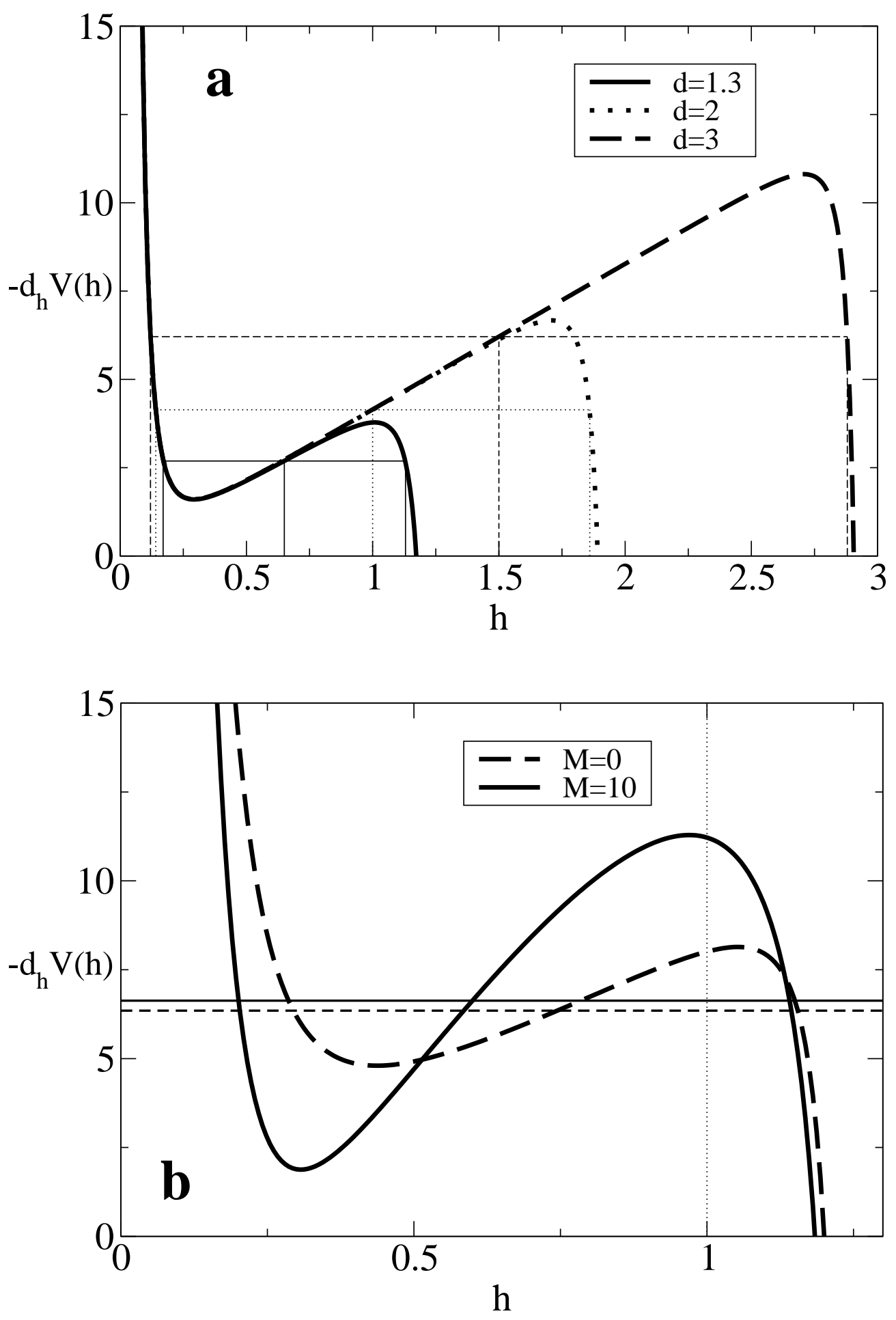

FIG. 7: D.Merkt, Physics of Fluids 


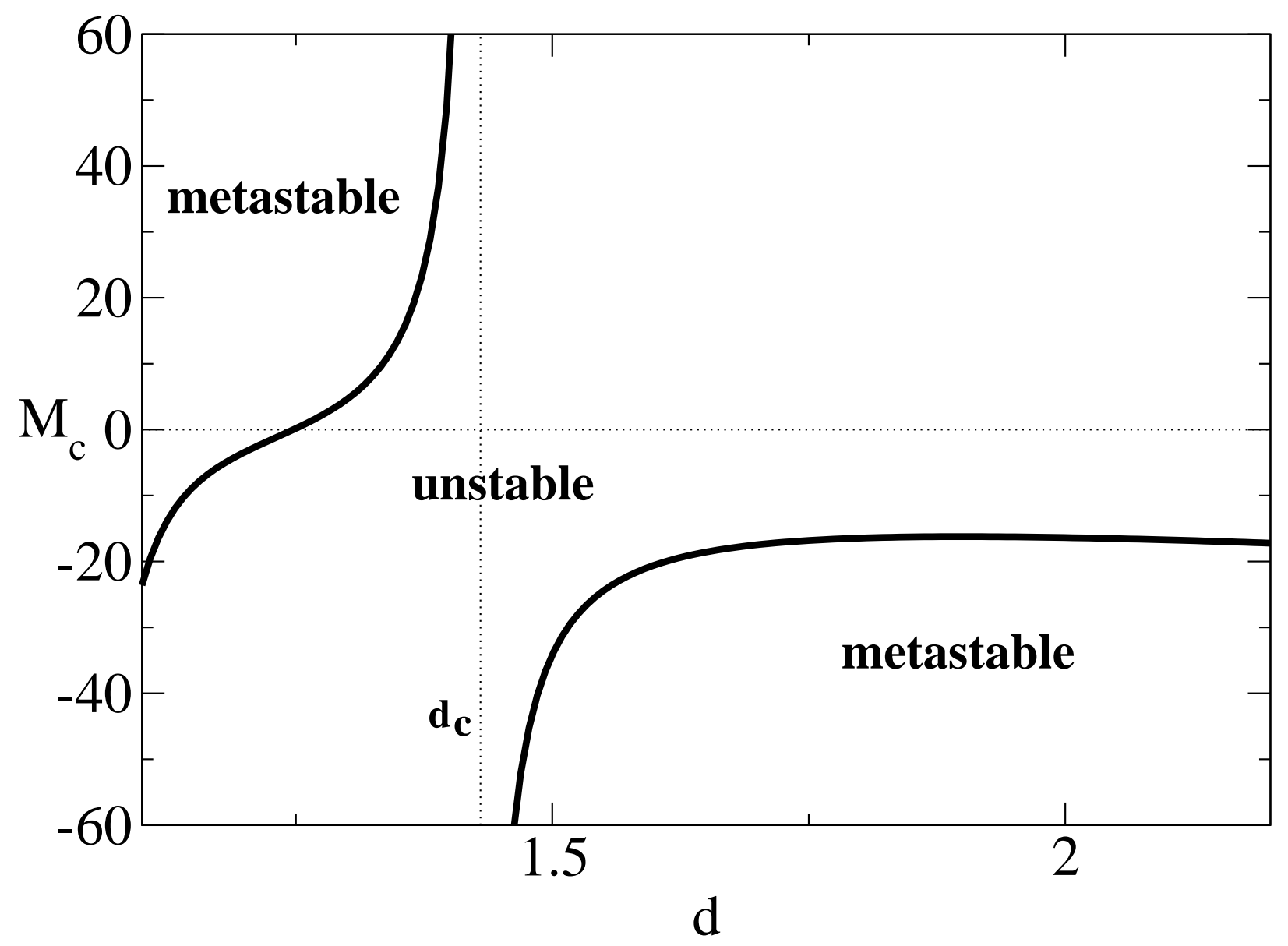

FIG. 8: D.Merkt, Physics of Fluids 


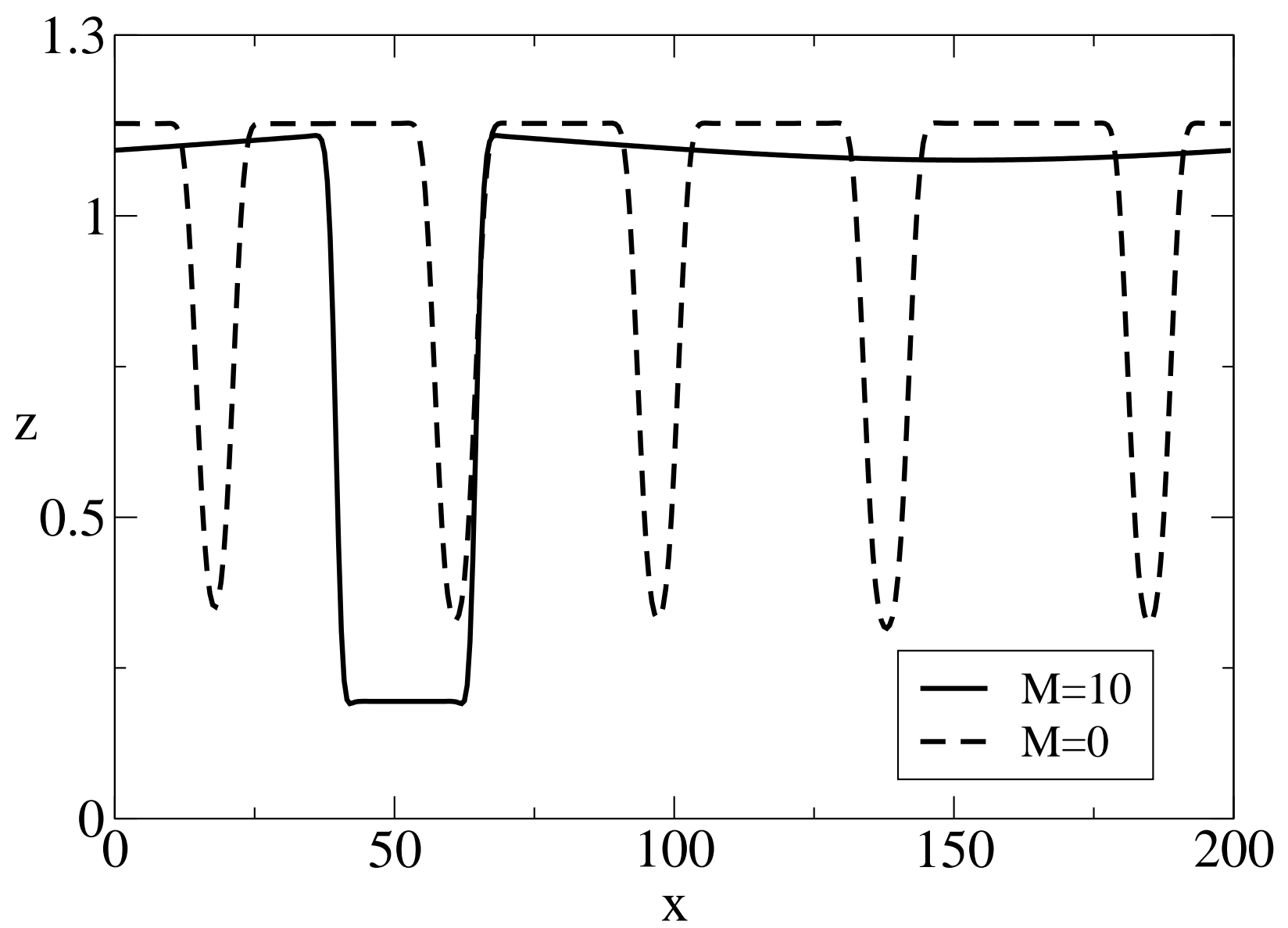

FIG. 9: D.Merkt, Physics of Fluids 


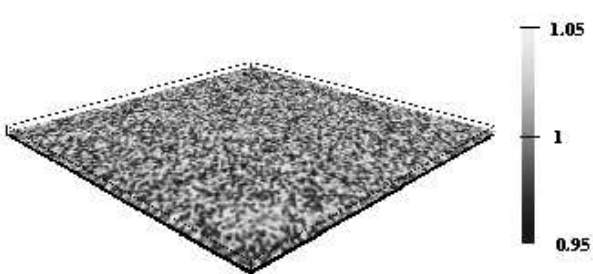

$\mathbf{t}=\mathbf{0}$

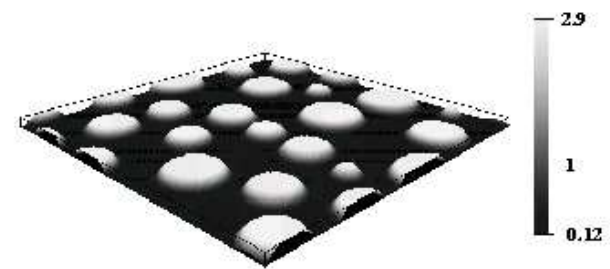

$t=100000$

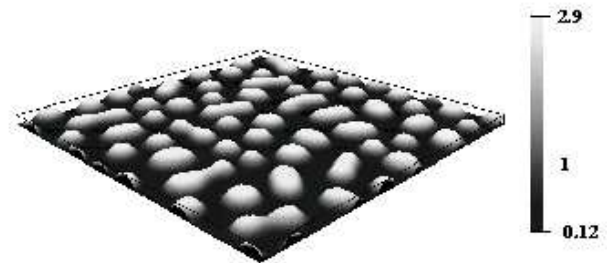

$t=1000$

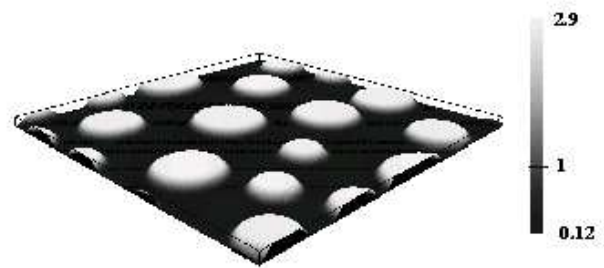

$\mathrm{t}=\mathbf{8 0 0 0 0 0}$

FIG. 10: D.Merkt, Physics of Fluids 

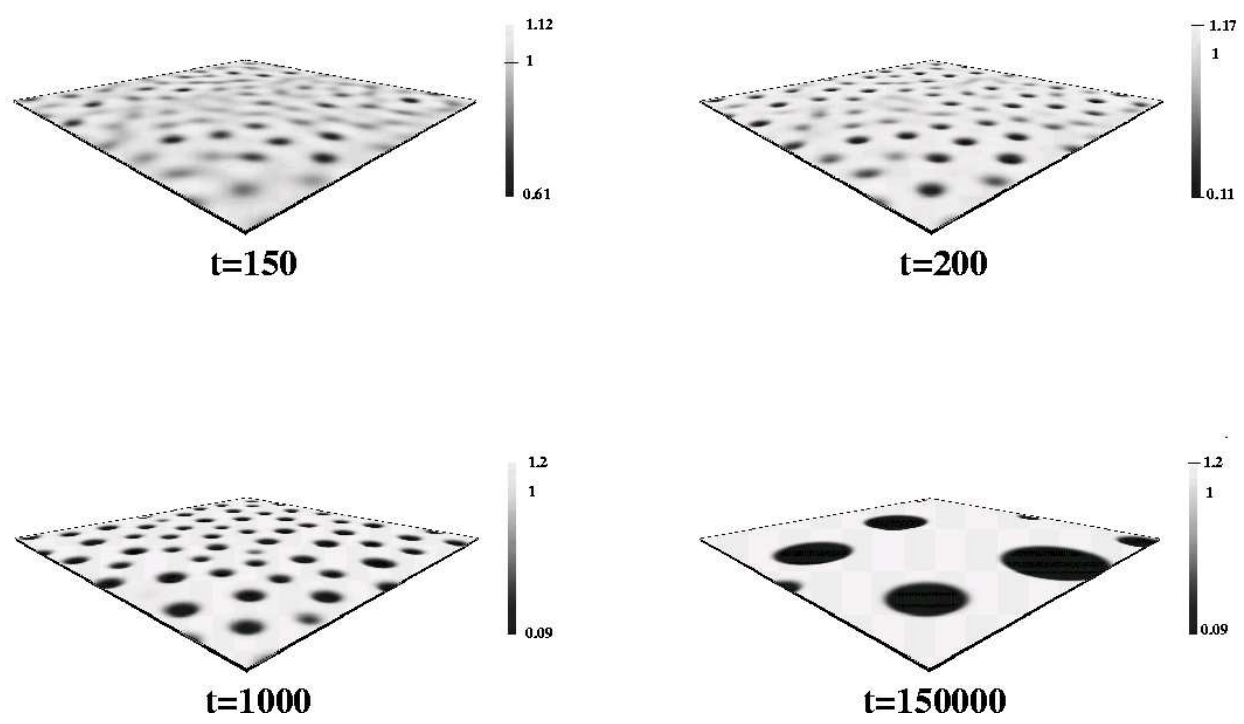

FIG. 11: D.Merkt, Physics of Fluids 


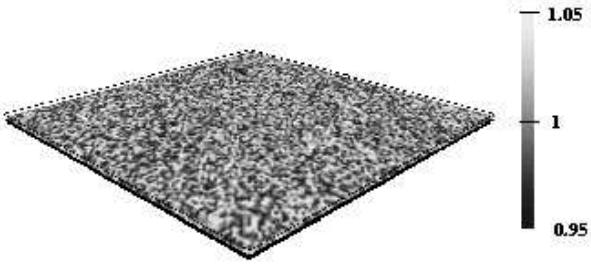

$\mathbf{t}=\mathbf{0}$

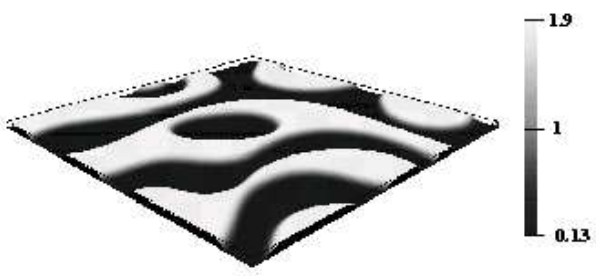

$\mathrm{t}=\mathbf{1 0 0 0 0 0}$
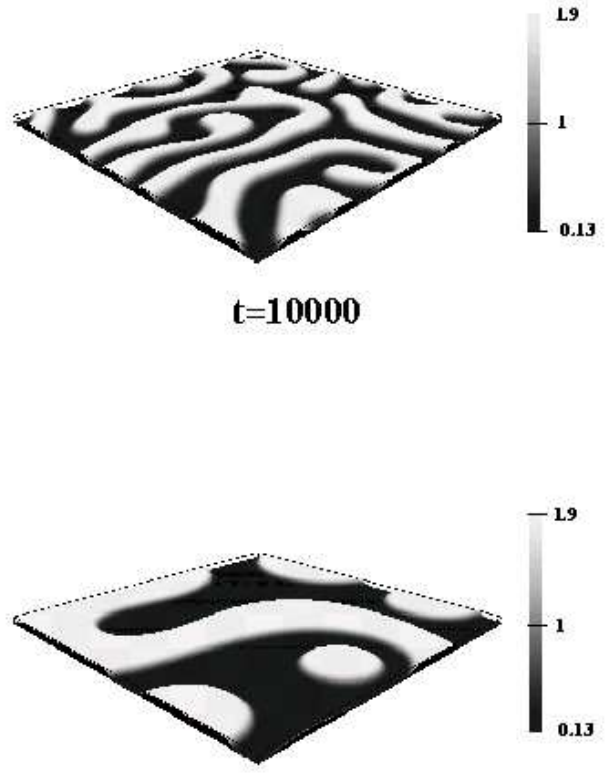

$t=150000$

FIG. 12: D.Merkt, Physics of Fluids 


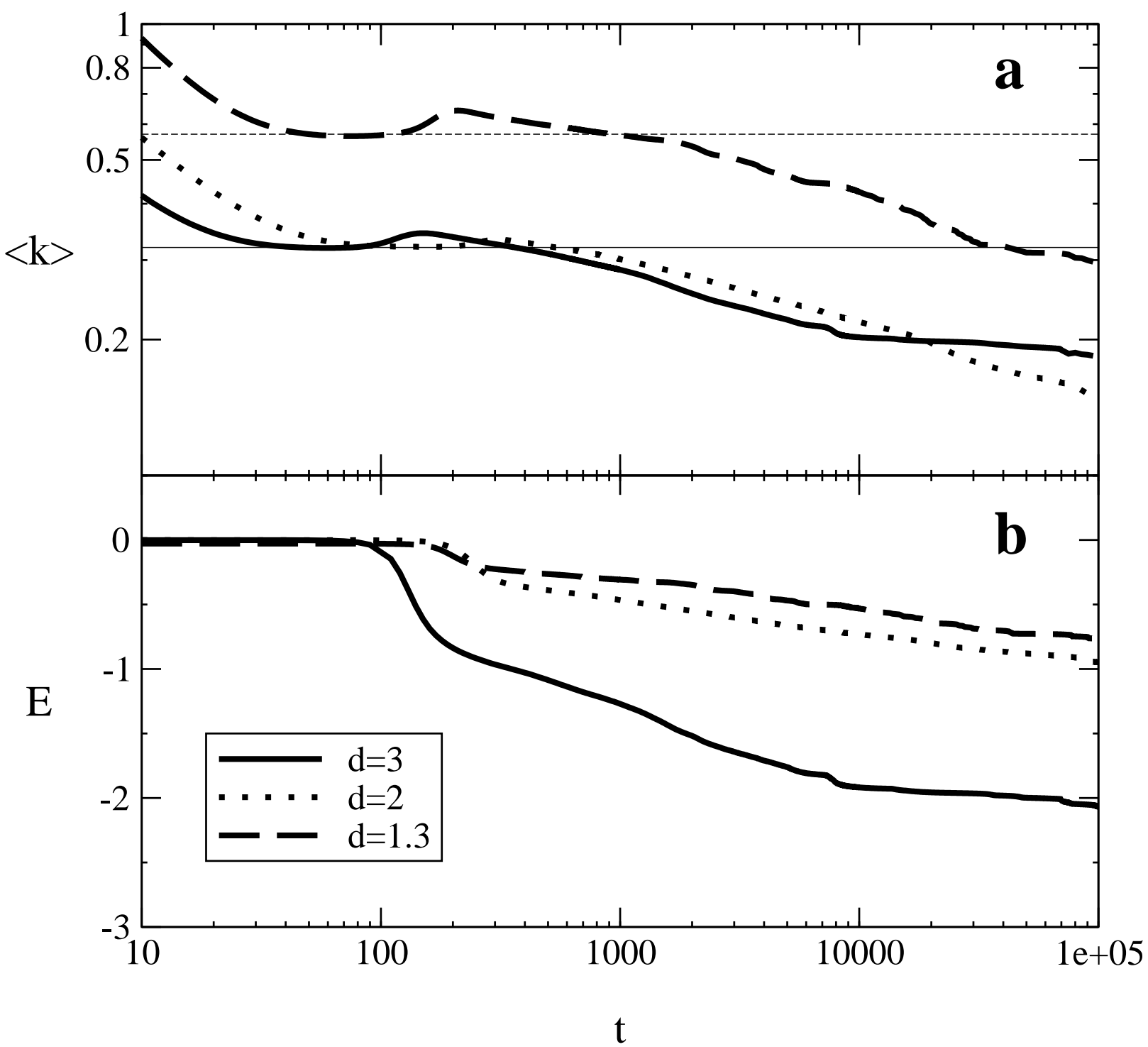

FIG. 13: D.Merkt, Physics of Fluids 
a) Translational mode

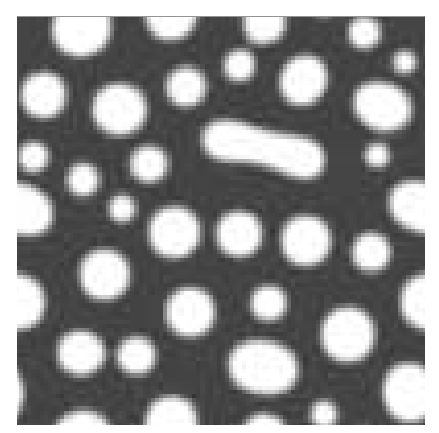

$\mathbf{t}=\mathbf{7 0 0 0}$

b) Volume transfer mode

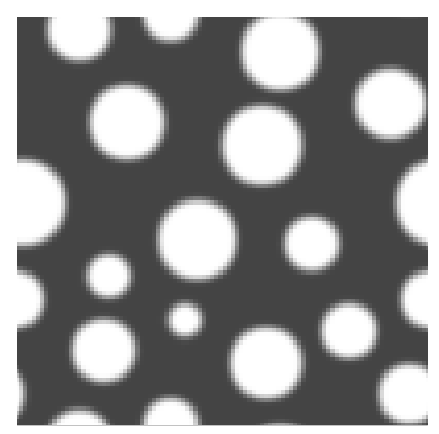

$t=39900$

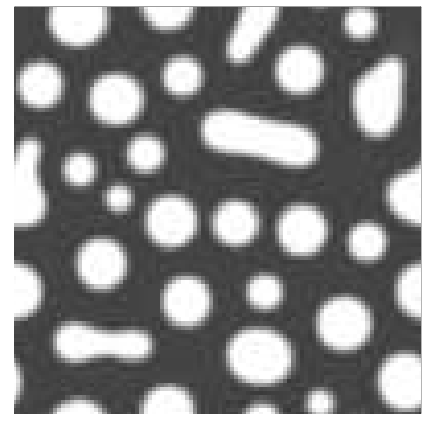

$t=8000$

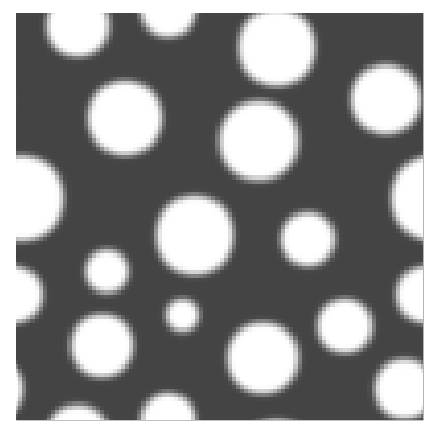

$t=40000$

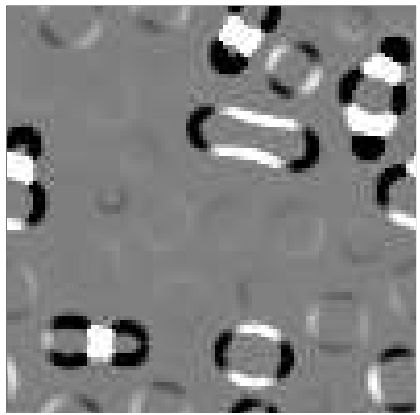

difference

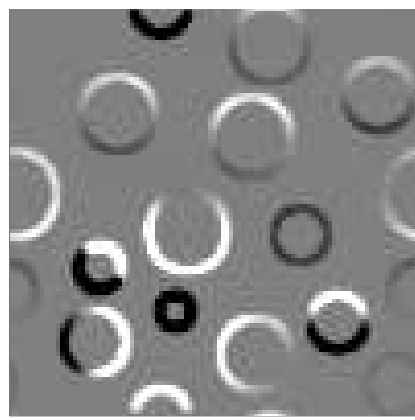

difference

FIG. 14: D.Merkt, Physics of Fluids 
a) $\mu<\mu_{\mathrm{c}}$
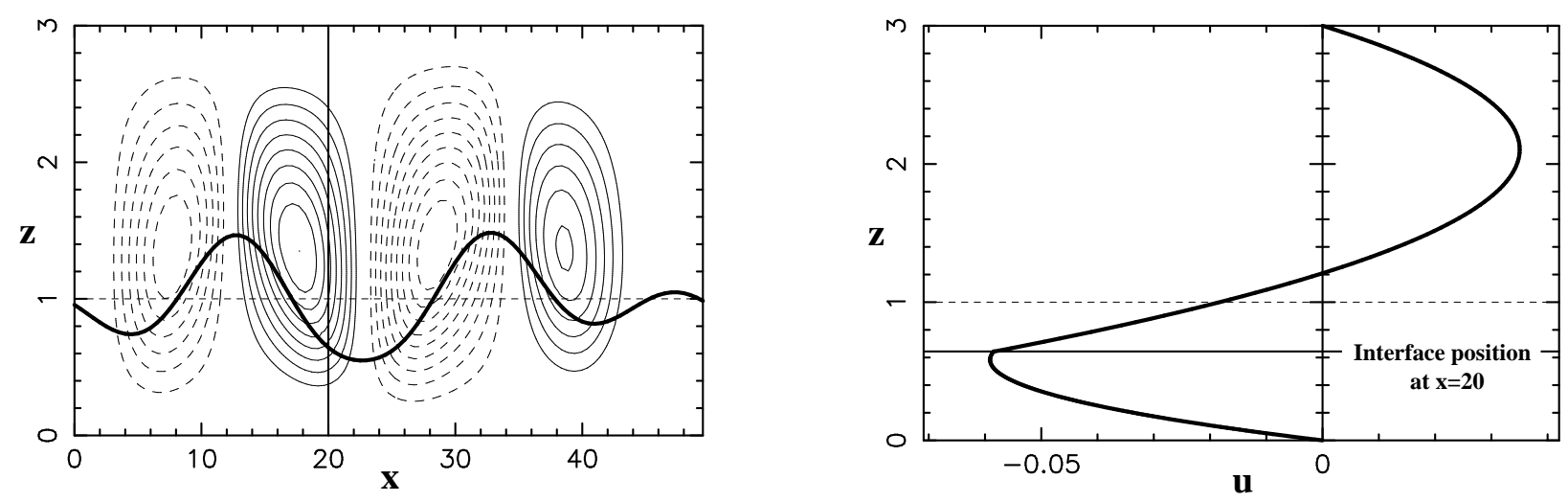

b) $\mu>\mu_{\mathrm{c}}$
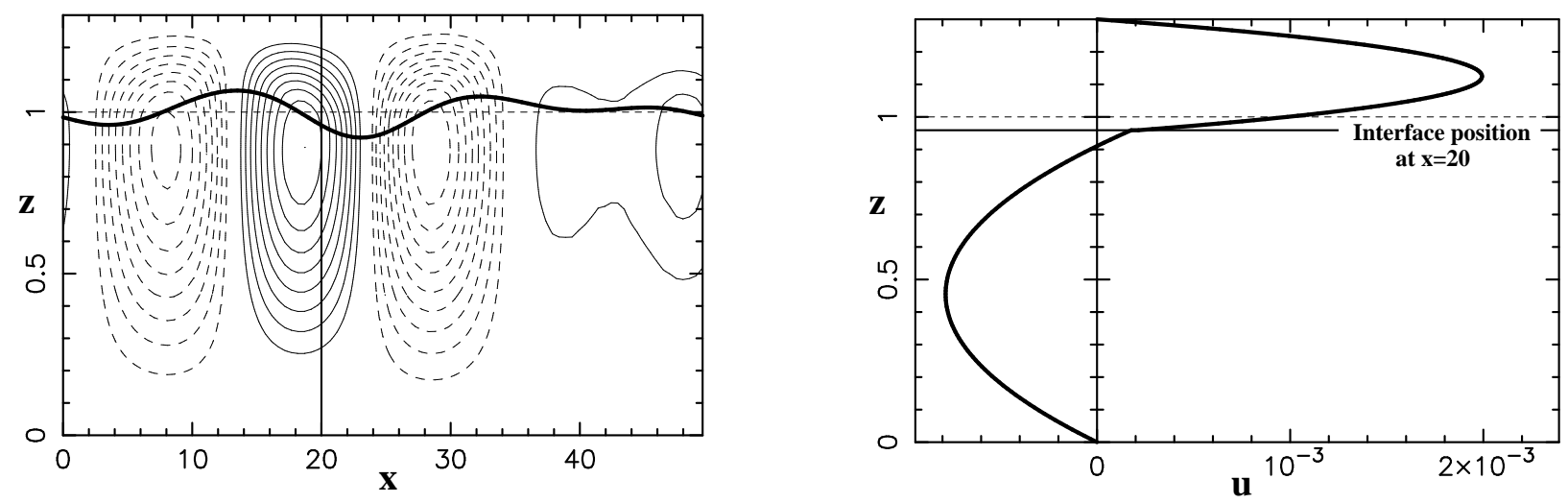

FIG. 15: D.Merkt, Physics of Fluids 

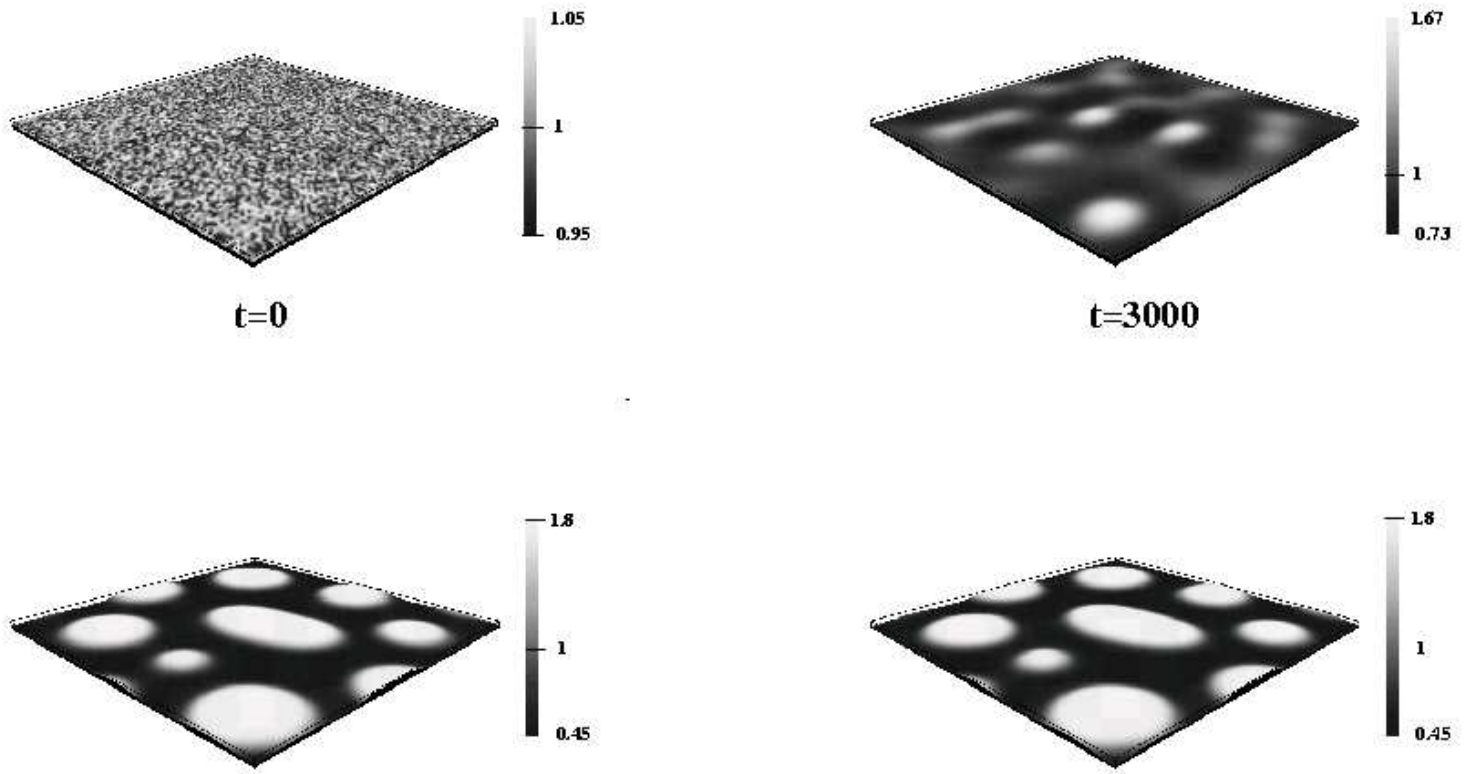

$t=30000$

$t=300000$

FIG. 16: D.Merkt, Physics of Fluids 


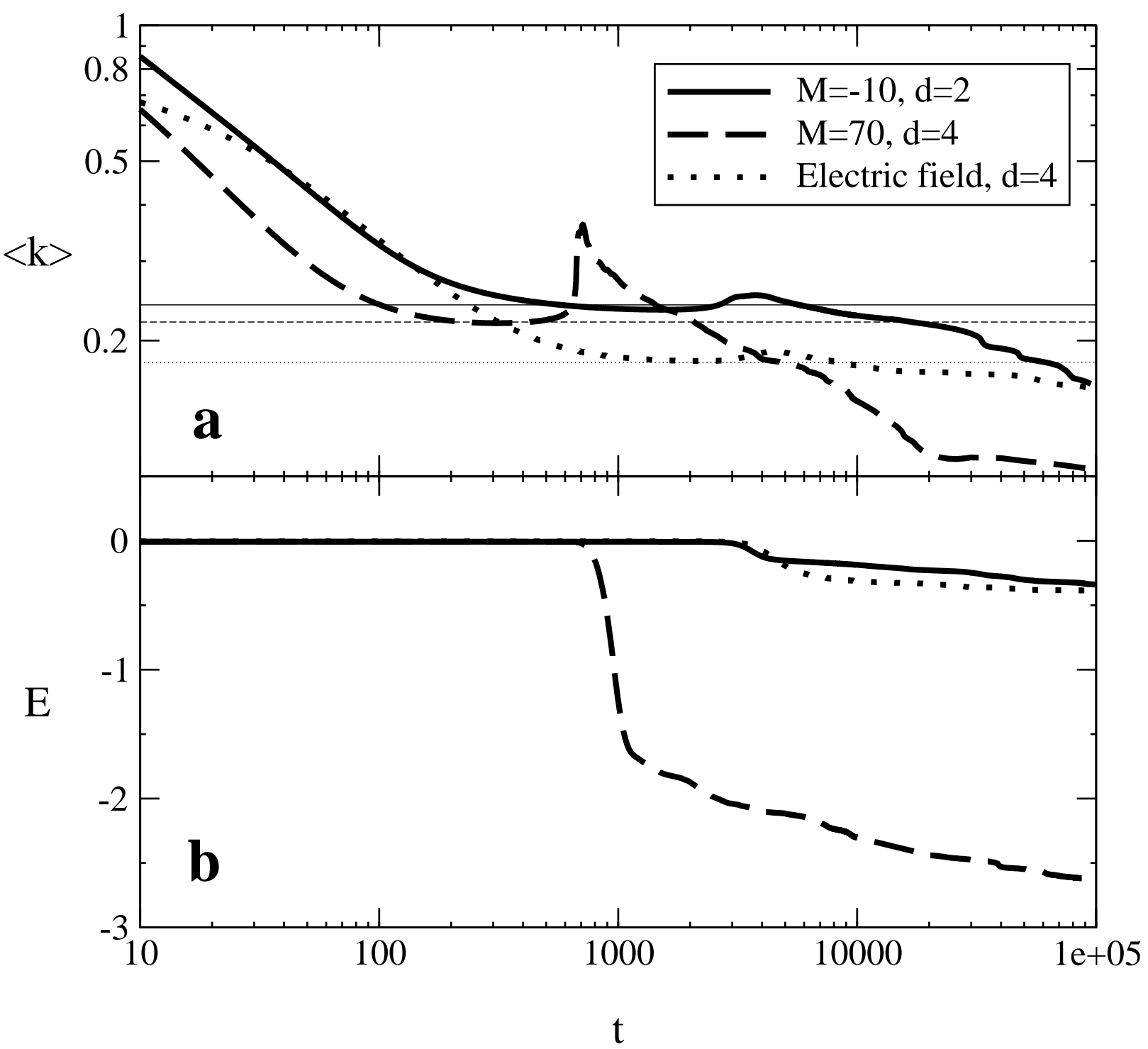

FIG. 17: D.Merkt, Physics of Fluids 

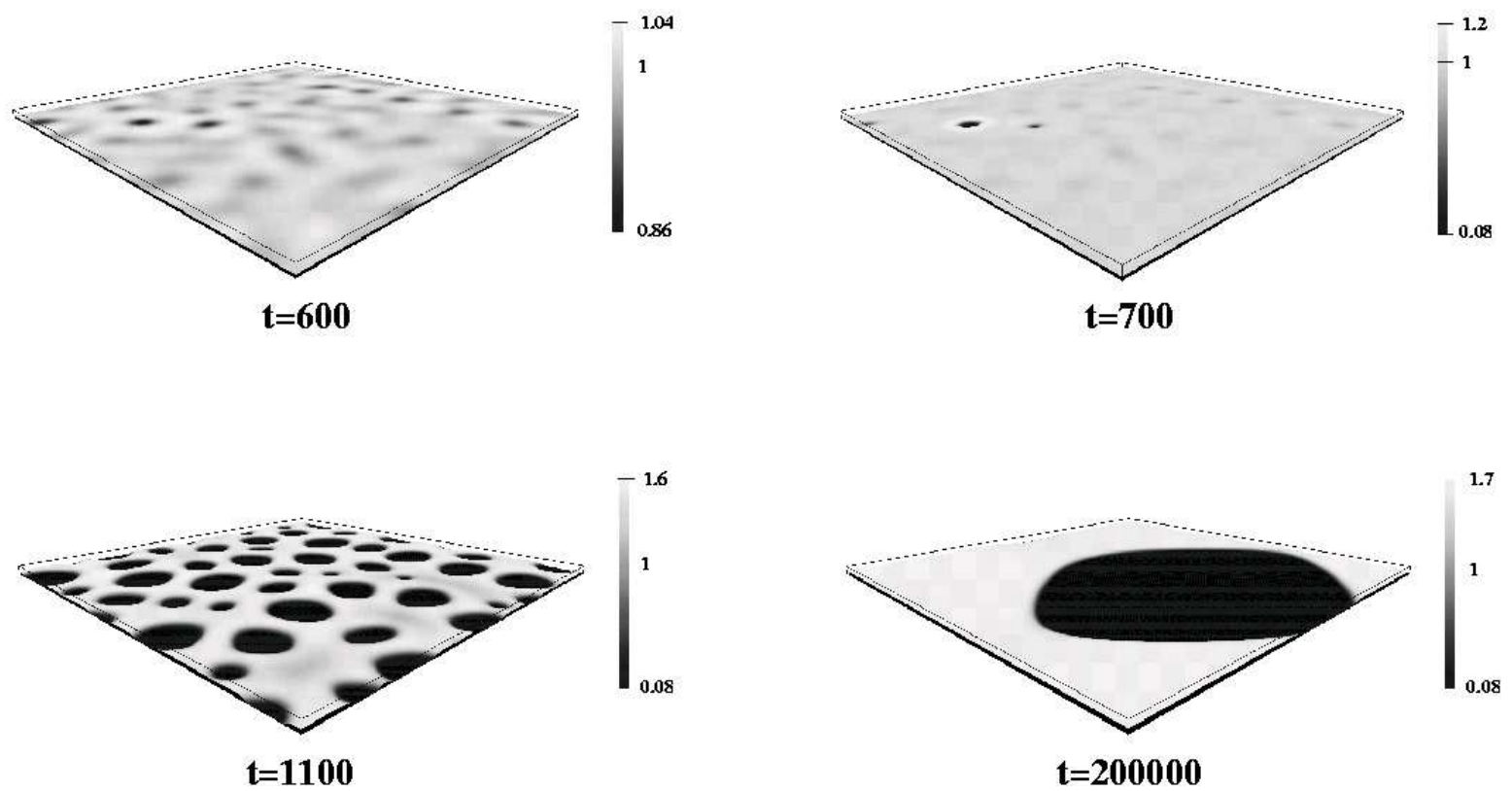

FIG. 18: D.Merkt, Physics of Fluids 


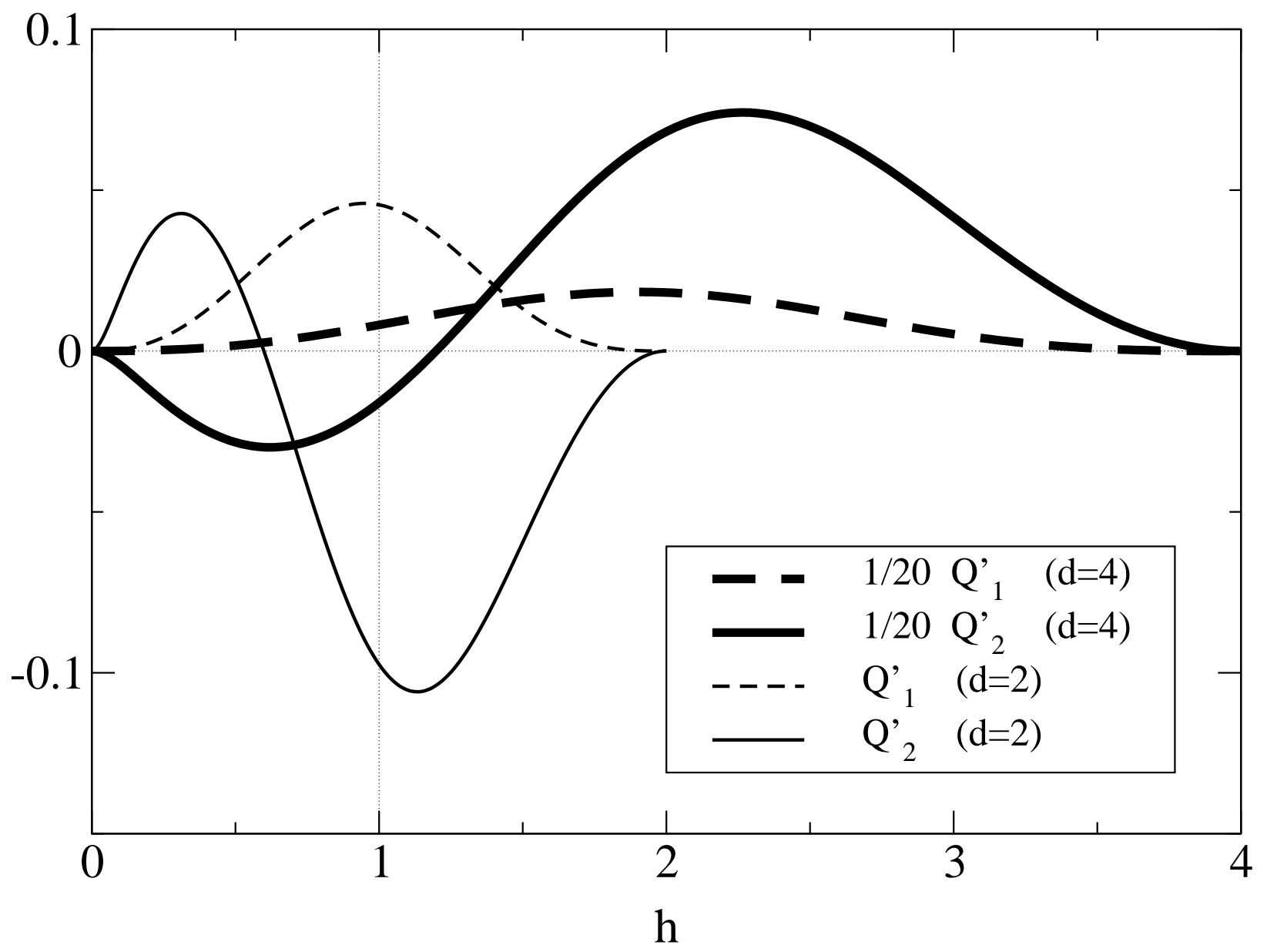

FIG. 19: D.Merkt, Physics of Fluids 


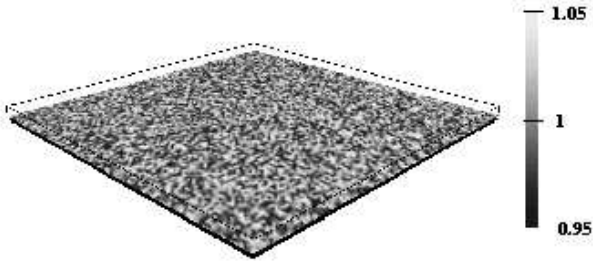

$\mathbf{t}=\mathbf{0}$

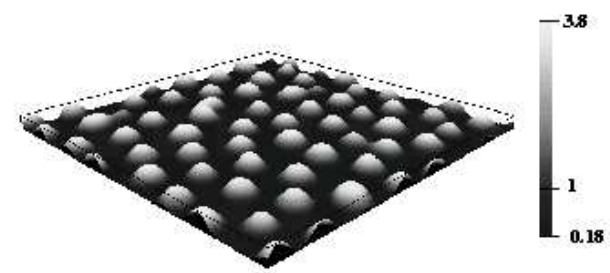

$t=10000$

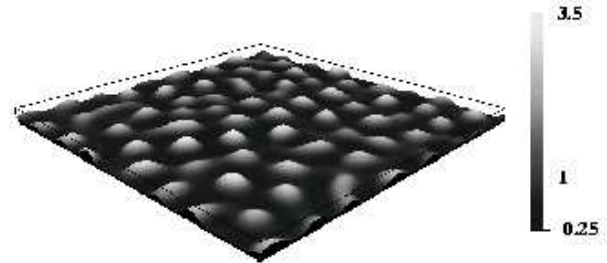

$t=4000$

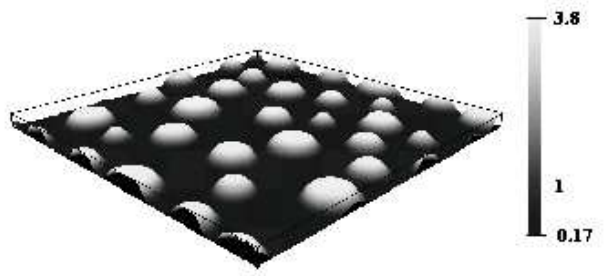

$\mathrm{t}=\mathbf{5 0 0 0 0 0}$

FIG. 20: D.Merkt, Physics of Fluids 